\title{
Modular functionals and perturbations of Nakano spaces
}

\author{
ITAÏ BEN YAACOV
}

\begin{abstract}
We settle several questions regarding the model theory of Nakano spaces left open by the $\mathrm{PhD}$ thesis of Pedro Poitevin [11].

We start by studying isometric Banach lattice embeddings of Nakano spaces, showing that in dimension two and above such embeddings have a particularly simple and rigid form.

We use this to show that in the Banach lattice language the modular functional is definable and that complete theories of atomless Nakano spaces are model complete. We also show that up to arbitrarily small perturbations of the exponent Nakano spaces are $\aleph_{0}$-categorical and $\aleph_{0}$-stable. In particular they are stable.
\end{abstract}

2000 Mathematics Subject Classification 03C98 (primary); 46E30 (secondary)

Keywords: Nakano space; continuous logic

\section{Introduction}

Nakano spaces are a generalisation of $L_{p}$ function spaces in which the exponent $p$ is allowed to vary as a measurable function of the underlying measure space. The $\mathrm{PhD}$ thesis of Pedro Poitevin [11] studies Nakano spaces as Banach lattices from a model theoretic standpoint. More specifically, he viewed Nakano spaces as continuous metric structures (in the sense of continuous logic, see [6]) in the language of Banach lattices, possibly augmented by a predicate symbol $\Theta$ for the modular functional, showed that natural classes of such structures are elementary in the sense of continuous first order logic, and studied properties of their theories.

In the present paper we propose to answer a few questions left open by Poitevin.

- First, Poitevin studies Nakano spaces in two natural languages: that of Banach lattices, and the same augmented with an additional predicate symbol for the modular functional. It is natural to ask whether these languages are truly distinct, i.e., whether adding the modular functional adds new structure. 
- Even if the naming of the modular functional does not add structure, it does give quantifier elimination in atomless Nakano spaces. While it is clear that without it quantifier elimination is impossible, it is natural to ask whether model completeness is.

- Poitevin showed that the theory of atomless Nakano space where the exponent function is bounded away from one is stable. What about the general case?

- Similarly, if the exponent is constant, i.e., if we are dealing with classical atomless $L_{p}$ spaces, it is known (see [4]) that the theory of these spaces is $\aleph_{0}$ categorical and $\aleph_{0}$-stable. On the other hand, it is quite easy to verify complete theories of atomless Nakano spaces are non $\aleph_{0}$-categorical and non $\aleph_{0}$-stable once the essential range of the exponent is infinite. It is therefore natural to ask whether, up to small perturbations of the exponent, a complete theory of atomless Nakano spaces is $\aleph_{0}$-categorical and $\aleph_{0}$-stable. A positive answer would mean that the theory of atomless Nakano spaces is stable settling the previous item as well.

In this paper we answer all of these questions positively (where a negative answer to the first question is considered positive). It is organised as follows:

Section 1 consist purely of functional analysis, and requires no familiarity with model theory. After a few general definitions we study mappings between vector lattices of measurable functions and then more specifically between Nakano spaces. Our main result is:

Theorem Let $\theta: L_{p(\cdot)}(X, \mathfrak{B}, \mu) \hookrightarrow L_{q(\cdot)}(Y, \mathfrak{C}, \nu)$ be a Banach lattice isometric embedding of Nakano spaces of dimension at least two. Then up to a measure density change on $Y$ and identification between subsets of $X$ and of $Y$ (and thus between measurable functions on $X$ and on $Y$ ) $\theta$ is merely an extension by zeros from $X$ to $Y \supseteq X$. In particular $p=\left.q\right|_{X}$ and $\mu=\nu \uparrow_{\mathfrak{B}}$.

It follows that such embeddings respect the modular functional and extend the essential range of the exponent function.

In Section 2 we expose the model theoretic setting for the paper. In particular, we quote the main results of Poitevin's PhD thesis [11].

In Section 3 we prove our main model theoretic results:

Theorem The modular functional is definable in every Nakano Banach lattice (i.e., naming it in the language does not add structure). Moreover, it is uniformly definable in the class of Nakano spaces of dimension at least two, and in fact both sup-definable and inf-definable there. 
Theorem The theory of atomless Nakano spaces with a fixed essential range for the exponent function is model complete in the Banach lattice language.

In Section 4 we study perturbations of the exponent function, showing that small perturbations thereof yield small perturbations of the structures. Up to such perturbations the theory of atomless Nakano spaces is $\aleph_{0}$-stable, and every completion thereof is $\aleph_{0}$-categorical. In particular all Nakano space are stable.

Appendix A consist of the adaptation to continuous logic of a few classical model theoretic results and tools used in this paper.

Finally, Appendix B contains some approximation results for the modular functional which were used in earlier versions of this paper to be superseded later by Theorem 1.10, but which nonetheless might be useful.

\section{Some functional analysis}

\subsection{Nakano spaces}

Let $(X, \mathfrak{B}, \mu)$ be an arbitrary measure space, and let $L_{0}(X, \mathfrak{B}, \mu)$ be the space of all measurable functions $f: X \rightarrow \mathbb{R}$ up to equality a.e. (Since we wish to consider function spaces as Banach lattices it will be easier to consider the case of real-valued functions.)

Let $p: X \rightarrow[1, \infty)$ be an essentially bounded measurable function. We define the modular functional $\Theta_{p(\cdot)}: L_{0}(X, \mathfrak{B}, \mu) \rightarrow[0, \infty]$ by:

$$
\Theta_{p(\cdot)}(f)=\int|f(x)|^{p(x)} d \mu .
$$

We define the corresponding Nakano space as:

$$
L_{p(\cdot)}(X, \mathfrak{B}, \mu)=\left\{f \in L_{0}(X, \mathfrak{B}, \mu): \Theta_{p(\cdot)}(f)<\infty\right\} .
$$

If $f \in L_{p(\cdot)}(X, \mathfrak{B}, \mu)$ then there exists a unique number $c \geq 0$ such that $\Theta_{p(\cdot)}(f / c)=1$, and we define $\|f\|=\|f\|_{p(\cdot)}=c$. This is a norm, making $L_{p(\cdot)}(X, \mathfrak{B}, \mu)$ a Banach space. With the point-wise minimum and maximum operations it is a Banach lattice.

Remark In the literature $\Theta_{p(\cdot)}$ is usually merely referred to as the modular. Being particularly sensitive regarding parts of speech we shall nonetheless refer to it throughout as the modular functional. 


\subsection{Strictly localisable spaces}

In this paper we shall consider the class of Nakano spaces from a model-theoretic point of view. This means we shall have to admit arbitrarily large Nakano spaces (e.g., $\kappa$-saturated for arbitrarily big $\kappa$ ) and therefore arbitrarily large measure spaces. In particular, we cannot restrict our attention to $\sigma$-finite measure spaces. In order to avoid pathologies which may arise with arbitrary measure spaces we shall require a weaker assumption. Recall from [7]:

Definition 1.1 A measure space $(X, \mathfrak{B}, \mu)$ is strictly localisable if it can be expressed as a disjoint union of measure spaces of finite measure, i.e., if $X$ admits a partition as $\bigcup_{i \in I} X_{i}$ such that:

(i) For all $i \in I: X_{i} \in \mathfrak{B}$ and $\mu\left(X_{i}\right)<\infty$.

(ii) For all $A \subseteq X$ : $A \in \mathfrak{B}$ if and only if $A \cap X_{i} \in \mathfrak{B}$ for all $i \in I$, in which case $\mu(A)=\sum \mu\left(A \cap X_{i}\right)$.

In this case the family $\left\{X_{i}\right\}_{i \in I}$ witnesses that $(X, \mathfrak{B}, \mu)$ is strictly localisable.

For example every $\sigma$-finite measure space is strictly localisable. On the other hand, if $(X, \mathfrak{B}, \mu)$ is an arbitrary measure space we can find a maximal family $\mathcal{X}=\left\{X_{i}\right\}_{i \in I} \subseteq \mathfrak{B}$ of almost disjoint sets with $0<\mu\left(X_{i}\right)<\infty$. Let $\left(X^{\prime}, \mathfrak{B}^{\prime}, \mu^{\prime}\right)=\coprod_{i \in I}\left(X_{i}, \mathfrak{B}\left\lceil_{X_{i}}, \mu \uparrow_{X_{i}}\right)\right.$, where the disjoint union of measure spaces is defined precisely so that the result is strictly localisable. We also have an obvious mapping $\theta: L_{0}(X, \mathfrak{B}, \mu) \rightarrow L_{0}\left(X^{\prime}, \mathfrak{B}^{\prime}, \mu^{\prime}\right)$. This does not lose any information that interests us: in particular, $\theta$ restricts to an isometric isomorphism of Nakano spaces $\theta: L_{p(\cdot)}(X, \mathfrak{B}, \mu) \rightarrow L_{\theta p(\cdot)}\left(X^{\prime}, \mathfrak{B}^{\prime}, \mu^{\prime}\right)$.

We may therefore allow ourselves:

Convention 1.2 In this paper every measure space is assumed to be strictly localisable.

Let us state a few very easy facts concerning strictly localisable measure spaces. The following is immediate:

Fact 1.3 Let $\mathcal{X}=\left\{X_{i}\right\}_{i \in I}$ witness that $(X, \mathfrak{B}, \mu)$ is strictly localisable. If $\mathcal{X}^{\prime}=$ $\left\{X_{j}^{\prime}\right\}_{j \in J} \subseteq \mathfrak{B}$ is another partition of $X$ refining $\mathcal{X}$, splitting each $X_{i}$ into at most countably many subsets, then $\mathcal{X}^{\prime}$ is a witness as well.

The Radon-Nikodým Theorem is classically stated for finite measure spaces, with various occurrences in the literature in which the finiteness requirement on the ambient 
space is relaxed. See for example [7, Corollaries 232F,G]. These are corollaries to [7, Theorem 232E], which allows an arbitrary ambient measure space at the cost of an additional concept, that of a truly continuous functional. Another generalisation appears in [8, Theorem 327D], but again the smaller measure is assumed there to be finite. We shall require a different generalisation of the Radon-Nikodým Theorem in which all finiteness requirements are replaced with strict localisability.

Let $(X, \mathfrak{B})$ be a measurable space and let $\mu$ and $\nu$ be two measures on $(X, \mathfrak{B})$. Assume also that $\nu(X)<\infty$. Then $\nu$ is said to be absolutely continuous with respect to $\mu$, in symbols $\nu \ll \mu$, if for every $\varepsilon>0$ there exists $\delta>0$ such that $\mu(A)<\delta \Longrightarrow \nu(A)<\varepsilon$ for every $A \in \mathfrak{B}$. Equivalently, if $\mu(A)=0 \Longrightarrow \nu(A)=0$ for every $A \in \mathfrak{B}$.

In the general case, i.e., when $\nu$ is not required to be finite, we shall use the notation $\nu \ll \mu$ to mean that $\mu$ and $\nu$ are both strictly localisable with a common witness $\left\{X_{i}\right\}_{i \in I}$, and that $\nu$ is absolutely continuous with respect to $\mu$ on each $X_{i}$. It follows directly from this definition that if $\nu \ll \mu$ and $\mu(A)=0$ for some $A \in \mathfrak{B}$ then $\nu(A)=0$ as well, so $\nu$ is absolutely continuous with respect to $\mu$ on every set of finite $\nu$-measure. This has two important consequences. First, if $\nu \ll \mu$ then every common witness of strict localisability for both $\mu$ and $\nu$ also witnesses that $\nu \ll \mu$. Second, in case $\nu(X)<\infty$, the definition of $\nu \ll \mu$ given in this paragraph coincides with the classical definition appearing in the previous paragraph.

Fact 1.4 Let $(X, \mathfrak{B}, \mu)$ be a measure space (strictly localisable, by our convention) and let $L_{0}^{+}(X, \mathfrak{B}, \mu)$ denote the set of positive functions in $L_{0}(X, \mathfrak{B}, \mu)$.

(i) Let $\zeta \in L_{0}^{+}(X, \mathfrak{B}, \mu)$, and for $A \in \mathfrak{B}$ define $\nu_{\zeta}(A)=\int \zeta d \mu$. Then $\nu_{\zeta}$ is a measure and $\nu_{\zeta} \ll \mu$.

(ii) Conversely, every measure $\nu \ll \mu$ on $(X, \mathfrak{B})$ is of the form $\nu=\nu_{\zeta}$ for a unique (up to equality $\mu$-a.e.) $\zeta \in L_{0}^{+}(X, \mathfrak{B}, \mu)$, and we write $\zeta=\frac{d \nu}{d \mu}$, the Radon-Nikodým derivative of $\nu$ with respect to $\mu$. In this case we also have $\int f d \nu=\int f \frac{d \nu}{d \mu} d \mu$ for every $f \in L_{0}^{+}(X, \mathfrak{B}, \mu)$.

In particular, we obtain a bijection between $\{\nu: \nu \ll \mu\}$ and $L_{0}^{+}(X, \mathfrak{B}, \mu)$.

Proof For the first item, let $\left\{X_{i}\right\}_{i \in I}$ witness that $\mu$ is strictly localisable. We may assume that in addition $\zeta$ is bounded on each $X_{i}$, for if not, we may split each $X_{i}$ into $X_{i, n}=\left\{x \in X_{i}: n \leq \zeta(x)<n+1\right\}$ for $n \in \mathbb{N}$. Then $\left\{X_{i}\right\}_{i \in I}$ also witnesses that $\nu_{\zeta}$ is strictly localisable and it is clear that $\nu_{\zeta} \ll \mu$.

For the converse, let $\left\{X_{i}\right\}_{i \in I}$ witness that $\nu \ll \mu$. We may apply the classical RadonNikodým theorem on each $X_{i}$, obtaining a measurable function $\zeta_{i}: X_{i} \rightarrow \mathbb{R}^{+}$for all 
$i \in I$, and define $\zeta: X \rightarrow \mathbb{R}^{+}$so that $\zeta\left\lceil_{X_{i}}=\zeta_{i}\right.$. Then $\zeta$ is measurable and

$$
\int f d \nu=\sum \int_{X_{i}} f d \nu=\sum \int_{X_{i}} f \zeta_{i} d \mu=\int f \zeta d \mu
$$

for $f \in L_{0}^{+}(X, \mathfrak{B}, \mu)$. In particular $\nu(A)=\int_{A} \zeta d \mu$ for all $A \in \mathfrak{B}$, which determines $\zeta$ up to equality $\mu$-a.e.

Let us say that two measures $\mu$ and $\nu$ on $(X, \mathfrak{B})$ are equivalent if $\mu \ll \nu$ and $\nu \ll \mu$. In this case each is obtained from the other by a mere density change and the corresponding Nakano spaces are naturally isomorphic.

Fact 1.5 Let $\mu$ and $\nu$ be two equivalent measures on $(X, \mathfrak{B})$, and let $p: X \rightarrow[1, r]$ be measurable. Let $(N, \Theta)=L_{p(\cdot)}(X, \mathfrak{B}, \mu)$ and $\left(N^{\prime}, \Theta^{\prime}\right)=L_{p(\cdot)}(X, \mathfrak{B}, \nu)$ be the corresponding Nakano spaces with their modular functionals. For $f \in N$ define $D_{\mu, \nu} f=\left(\frac{d \mu}{d \nu}\right)^{1 / p} f$. Then $D_{\mu, \nu} f \in N^{\prime}$ and $D_{\mu, \nu}:(N, \Theta) \simeq\left(N^{\prime}, \Theta^{\prime}\right)$ is an (isometric) isomorphism.

Proof One calculates:

$$
\begin{aligned}
\Theta^{\prime}\left(D_{\mu, \nu} f\right) & =\int\left(\left(\frac{d \mu}{d \nu}\right)^{1 / p}|f|\right)^{p} d \nu \\
& =\int|f|^{p} \frac{d \mu}{d \nu} d \nu \\
& =\int|f|^{p} d \mu=\Theta(f) .
\end{aligned}
$$

It follows that $f \in N \Longrightarrow D_{\mu, \nu} f \in N^{\prime}$. In addition to $\Theta, D_{\mu, \nu}$ clearly also respects the linear and lattice structures, and therefore the norm, and admits an inverse $D_{\nu, \mu}$.

\subsection{Mappings between function space lattices}

For the following results we shall be considering two measure spaces $(X, \mathfrak{B}, \mu)$ and $(Y, \mathfrak{C}, \nu)$, as well as a partial mapping $\theta: L_{0}(X, \mathfrak{B}, \mu) \rightarrow L_{0}(Y, \mathfrak{C}, \nu)$. Its domain $L \subseteq L_{0}(X, \mathfrak{B}, \mu)$ is a vector subspace which contains all characteristic functions of finite measure sets. For example, $L$ could be a Nakano space $L_{p(\cdot)}(X, \mathfrak{B}, \mu)$ or just the space of simple functions on $X$ with finite measure support. Assuming that $\theta$ sends characteristic functions to characteristic functions, we shall allow ourselves the following abuse of notation: if $A \in \mathfrak{B}$ has finite measure and $\theta\left(\chi_{A}\right)=\chi_{B}, B \in \mathfrak{C}$ then we write $\theta A=B$ (even though this is only defined up to null measure). In particular, instead of writing $\theta\left(\chi_{A}\right)$ we write $\chi_{\theta A}$. 
Lemma 1.6 Let $L \subseteq L_{0}(X, \mathfrak{B}, \mu)$ be a vector subspace which contains all characteristic functions of finite measure sets and let $\theta: L \rightarrow L_{0}(Y, \mathfrak{C}, \nu)$ be a linear mapping respecting point-wise countable suprema when those exist in $L$, and which in addition sends characteristic functions to characteristic functions.

Then $\theta$ extends to a unique vector lattice homomorphism $\hat{\theta}: L_{0}(X, \mathfrak{B}, \mu) \rightarrow L_{0}(Y, \mathfrak{C}, \nu)$ which respects countable suprema. Moreover, for every Borel function $\varphi: \mathbb{R}^{n} \rightarrow \mathbb{R}$ which fixes zero (i.e., which sends $0 \in \mathbb{R}^{n}$ to $0 \in \mathbb{R}$ ) and every tuple $\bar{f} \in L_{0}(X, \mathfrak{B}, \mu)$ we have $\hat{\theta}(\varphi \circ \bar{f})=\varphi \circ(\theta \bar{f})$.

Proof Let us write $L_{0}$ for $L_{0}(X, \mathfrak{B}, \mu)$, and let $L_{0}^{+}$be its positive cone.

Let us first consider the case where $\mu(X), \nu(Y)<\infty$. In this case $L$ contains all simple measurable functions. For $f \in L_{0}^{+}$and $0<k \in \mathbb{N}$ define $f^{(k)}(x)=\frac{\lceil k f(x)\rceil}{k+1} \wedge k$, where $\lceil r\rceil$ denotes the least integer greater than $r$. Thus $f^{(k)} \nearrow f$ point-wise and $f^{(k)} \in L$ for all $k$. We then have no choice but to define $\hat{\theta}$ as follows:

$$
\begin{array}{ll}
\hat{\theta} f=\hat{\theta}\left(\bigvee_{k \in \mathbb{N}} f^{(k)}\right)=\bigvee_{k \in \mathbb{N}} \theta f^{(k)} & \text { for } f \in L_{0}^{+}, \\
\hat{\theta} f=\hat{\theta}\left(f^{+}-f^{-}\right)=\hat{\theta} f^{+}-\hat{\theta} f^{-} & \text {for general } f \in L_{0} .
\end{array}
$$

We now need to make sure this verifies all the requirements.

First of all we need to check that if $f \in L_{0}^{+}$then $\hat{\theta} f=\bigvee_{k \in \mathbb{N}} \theta f^{(k)}$ exists, i.e., that it is finite a.e. Let $A_{k}=\{f \geq k\}=\{x \in X: f(x) \geq k\}$. Then the sequence $\left\{\chi_{A_{k}}\right\}$ decreases to zero, whereby $\left\{\chi_{\theta A_{k}}\right\}$ decrease to zero as well. We have $f^{(k+m)} \leq k+m \chi_{A_{k}}$ whereby $\theta f^{(k+m)} \leq k+m \chi_{\theta A_{k}}$, so $\theta f^{(k+m)} \leq k$ outside $\theta A_{k}$, for all $m$. Thus $\hat{\theta} f \leq k$ outside $\theta A_{k}$, and we can conclude that $\hat{\theta} f \in L_{0}(Y, \mathfrak{C}, \nu)$. Since $\theta$ respects countable suprema, $\hat{\theta}$ agrees with $\theta$ on $L^{+}$.

We claim that $\hat{\theta}$ respects countable suprema on $L_{0}^{+}$. Indeed, assume that $\bigvee_{m \in \mathbb{N}} f_{m}$ exists for $f_{m} \in L_{0}^{+}$. Notice that in general $\bigvee_{m}\left\lceil a_{m}\right\rceil=\left\lceil\bigvee_{m} a_{m}\right\rceil$, whereby

$\hat{\theta}\left(\bigvee_{m \in \mathbb{N}} f_{m}\right)=\bigvee_{k \in \mathbb{N}} \theta\left(\left(\bigvee_{m \in \mathbb{N}} f_{m}\right)^{(k)}\right)=\bigvee_{k \in \mathbb{N}} \theta\left(\bigvee_{m \in \mathbb{N}} f_{m}^{(k)}\right)=\bigvee_{m \in \mathbb{N}, k \in \mathbb{N}} \theta\left(f_{m}^{(k)}\right)=\bigvee_{m \in \mathbb{N}} \hat{\theta}\left(f_{m}\right)$.

If $f=\sum_{m \in \mathbb{N}} f_{m}$ where $f_{m} \in L_{0}^{+}, f_{m} \wedge f_{m^{\prime}}=0$ for $m \neq m^{\prime}$ then $\theta\left(f_{m}\right) \wedge \theta\left(f_{m^{\prime}}\right)=0$ as well and $\hat{\theta}(f)=\hat{\theta}\left(\bigvee_{m} f_{m}\right)=\bigvee_{m} \hat{\theta}\left(f_{m}\right)=\sum_{m} \hat{\theta}\left(f_{m}\right)$.

Next we claim that if $A \subseteq(0, \infty)^{n}$ is a Borel set and $\bar{f} \in\left(L_{0}^{+}\right)^{n}$ then $\theta\{\bar{f}(x) \in$ $A\}=\{\hat{\theta} \bar{f}(y) \in A\}$. Indeed, for a single $f$ we have $\hat{\theta} f=\hat{\theta}\left(\bigvee_{t \in \mathbb{Q}^{+}} t \chi_{\{f>t\}}\right)=$ 
$\bigvee_{t \in \mathbb{Q}^{+}} t \chi_{\theta\{f>t\}}$, whereby $\{\hat{\theta} f>t\}=\theta\{f>t\}$. Our claim follows for the case $A=\left(t_{0}, \infty\right) \times \ldots \times\left(t_{n-1}, \infty\right)$. On the other hand we have

$$
\begin{gathered}
\theta\left\{\bar{f}(x) \in \bigcup_{m} A_{m}\right\}=\bigcup_{m} \theta\left\{\bar{f}(x) \in A_{m}\right\}, \\
\left\{\hat{\theta} \bar{f}(y) \in \bigcup_{m} A_{m}\right\}=\bigcup_{m}\left\{\hat{\theta} \bar{f}(y) \in A_{m}\right\}, \\
\theta\left\{\bar{f}(x) \in(0, \infty)^{n} \backslash A\right\}=\theta\{\bar{f}>0\} \backslash \theta\{\bar{f}(x) \in A\}, \\
\left\{\hat{\theta} \bar{f}(y) \in(0, \infty)^{n} \backslash A\right\}=\theta\{\bar{f}>0\} \backslash\{\hat{\theta} \bar{f}(y) \in A\} .
\end{gathered}
$$

We may thus climb up the Borel hierarchy and prove the claim for all Borel $A$.

Assume now that $\bar{f}(x) \in(0, \infty)^{n} \cup\{0\}$ for all $x \in X$ and that $\varphi \geq 0$. Letting $A_{t}=\left\{x \in(0, \infty)^{n}: \varphi(x)>t\right\}$ :

$$
\theta\{\varphi \circ \bar{f}>t\}=\theta\left\{\bar{f} \in A_{t}\right\}=\left\{\hat{\theta} \bar{f} \in A_{t}\right\}=\{\varphi \circ(\hat{\theta} \bar{f})>t\},
$$

whereby $\hat{\theta}(\varphi \circ \bar{f})=\varphi \circ(\hat{\theta} \bar{f})$. For general $\bar{f}$, let $S=\{1,0,-1\}^{n} \backslash\{0\}$, and for $s \in S$ let $A_{s}=\{x \in X: \operatorname{sgn}(\bar{f})=s\}$. On each $A_{s}$ we may drop those $f_{i}$ 's which are constantly zero and replace those which are negative with their absolute value, making the necessary modifications to $\varphi$, obtaining by the previous argument

$$
\hat{\theta}\left(\varphi \circ\left(\chi_{A_{s}} \bar{f}\right)\right)=\varphi \circ\left(\hat{\theta}\left(\chi_{A_{s}} \bar{f}\right)\right)
$$

whereby:

$$
\hat{\theta}(\varphi \circ \bar{f})=\sum_{s \in S} \hat{\theta}\left(\varphi \circ\left(\chi_{A_{s}} \bar{f}\right)\right)=\sum_{s \in S} \varphi \circ\left(\hat{\theta}\left(\chi_{A_{s}} \bar{f}\right)\right)=\varphi \circ(\hat{\theta} \bar{f})
$$

Finally, for general $\varphi$ we can split it to the positive and negative part and then put them back together by linearity. Among other things, this holds when $\varphi$ is $+, \vee, \wedge$, or multiplication by scalar. Thus $\hat{\theta}$ is a vector lattice homomorphism. It follows that $\hat{\theta}$ agrees with $\theta$ on all of $L$. This concludes the case where both $X$ and $Y$ have finite total measure.

Now let us consider the case where $X$ is an arbitrary measure space. Let $\left\{X_{i}\right\}_{i \in I} \subseteq \mathfrak{B}$ be a maximal family of almost disjoint sets of finite non zero measure such that in addition $\theta\left(\chi_{X_{i}}\right) \neq 0$. Since $\nu(Y)$ is assumed finite such a family must be at most countable, so we can write it as $\left\{X_{k}\right\}_{k \in \mathbb{N}}$. Let $X^{\prime}=\bigcup X_{k}$. Then for every $f \in L$ we have $\theta(f)=\theta\left(f \chi_{X^{\prime}}\right)=\sum_{k} \theta\left(f \chi_{X_{k}}\right)$ (verify first for $f \geq 0$ and then extend by linearity), so we may restrict to each $X_{k}$, reducing to the case already considered, then checking that $\hat{\theta}(f)=\sum_{k} \hat{\theta}\left(f \chi_{X_{k}}\right)$ works. 
Finally, if $(Y, \mathfrak{C}, \nu)$ is merely strictly localisable then let this be witnessed by $\left\{Y_{i}\right\}_{i \in I}$. Then we can first extend $\theta_{i}=\chi_{Y_{i}} \theta: L \rightarrow\left(Y_{i}, \mathfrak{C}_{Y_{Y_{i}}}, \nu \uparrow_{Y_{i}}\right)$ to $\hat{\theta}_{i}$ and then obtain $\hat{\theta}$ by gluing.

Lemma 1.7 Continue with previous assumptions, and add that if $\mu(A)<\infty$ then $\nu(\theta A)=\mu(A)$. Then for every function $f \in L_{1}(X, \mathfrak{B}, \mu): \int f d \mu=\int \hat{\theta} f d \nu$.

Proof This holds by assumption for characteristic functions of finite measure sets, from which we deduce it for simple positive functions, positive functions and finally general functions.

\subsection{Embeddings of Nakano Banach lattices}

We now prove the main functional analysis results of this paper.

Lemma 1.8 Let $N=L_{p(\cdot)}(X, \mathfrak{B}, \mu)$ and $N^{\prime}=L_{q(\cdot)}(Y, \mathfrak{C}, \nu)$ be two Nakano spaces, and let $\theta: N \rightarrow N^{\prime}$ be an isometric embedding of Banach lattices which sends characteristic functions to characteristic functions. Assume furthermore that $\operatorname{dim} N \geq 2$.

Then:

(i) $\hat{\theta}(p)=q \chi_{\hat{\theta} X}$.

(ii) For all finite measure $A \in \mathfrak{B}: \nu(\theta A)=\mu(A)$.

Proof First of all the hypotheses of Lemma 1.6 are verified with $N=L$, so $\hat{\theta}$ exists. Let $Y_{0}=\hat{\theta} X \in \mathfrak{C}$ be the support of the range of $\theta$.

$$
\begin{aligned}
C_{1} & =\left\{y \in Y_{0}: \hat{\theta} p(y)<q(y)\right\}, \\
C_{2} & =\left\{y \in Y_{0}: \hat{\theta} p(y)>q(y)\right\}, \\
C & =C_{1} \cup C_{2}=\left\{y \in Y_{0}: \hat{\theta} p(y) \neq q(y)\right\}
\end{aligned}
$$

Then $C_{1}, C_{2}, C \in \mathfrak{C}$ and we need to show that $\nu(C)=0$. Let $A, B \in \mathfrak{B}$ be such that $0<\mu(A), \mu(B)<\infty$. For $t \in[0,1]$, let

$$
\begin{gathered}
f_{t}=\chi_{A}\left(\frac{t}{\mu(A)}\right)^{\frac{1}{p(x)}}+\chi_{B}\left(\frac{1-t}{\mu(B)}\right)^{\frac{1}{p(x)}} \\
g_{t}=\theta\left(f_{t}\right)=\chi_{\theta A}\left(\frac{t}{\mu(A)}\right)^{\frac{1}{\hat{\theta}(y)}}+\chi_{\theta B}\left(\frac{1-t}{\mu(B)}\right)^{\frac{1}{\hat{\theta}(y)}},
\end{gathered}
$$


Then $\Theta\left(f_{t}\right)=1 \Longrightarrow\left\|f_{t}\right\|=1 \Longrightarrow\left\|g_{t}\right\|=1 \Longrightarrow \Theta^{\prime}\left(g_{t}\right)=1$. In other words:

$$
\Theta^{\prime}\left(g_{t}\right)=\int_{\theta A}\left(\frac{t}{\mu(A)}\right)^{\frac{q}{\hat{\theta}_{p}}} d \nu+\int_{\theta B}\left(\frac{1-t}{\mu(B)}\right)^{\frac{q}{\hat{\theta}_{p}}} d \nu=1
$$

Substituting $t=0$ and $t=1$ we see that in particular $\nu(A)$ and $\nu(B)$ are both positive and finite. We may therefore differentiate under the integral sign for $t \in(0,1)$, obtaining:

$$
\begin{aligned}
0=\frac{d}{d t} \Theta^{\prime}\left(g_{t}\right)= & \int_{\theta A \cap C} \frac{q}{\mu(A) \hat{\theta} p}\left(\frac{t}{\mu(A)}\right)^{\frac{q}{\hat{\theta} p}-1} d \nu+\int_{\theta A \backslash C} \frac{q}{\mu(A) \hat{\theta} p} d \nu \\
& -\int_{\theta B \cap C} \frac{q}{\mu(B) \hat{\theta} p}\left(\frac{1-t}{\mu(B)}\right)^{\frac{q}{\hat{\theta} p}-1} d \nu-\int_{\theta B \backslash C} \frac{q}{\mu(B) \hat{\theta} p} d \nu
\end{aligned}
$$

If $\nu\left(\theta A \cap C_{2}\right)>0$ then $\lim _{t \rightarrow 0} \frac{d}{d t} \Theta^{\prime}\left(g_{t}\right)=+\infty \neq 0$ which is impossible, so $\nu(\theta A \cap$ $\left.C_{2}\right)=0$, and considering $t \rightarrow 1$ we see that $\nu\left(\theta B \cap C_{2}\right)=0$ as well. We may therefore substitute $t=0$ and $t=1$ and obtain:

$$
\begin{aligned}
0= & \int_{\theta A \backslash C} \frac{q}{\mu(A) \hat{\theta} p} d \nu \\
& -\int_{\theta B \cap C} \frac{q}{\mu(B) \hat{\theta} p}\left(\frac{1}{\mu(B)}\right)^{\frac{q}{\theta_{p}}-1} d \nu-\int_{\theta B \backslash C} \frac{q}{\mu(B) \hat{\theta} p} d \nu \\
= & \int_{\theta A \cap C} \frac{q}{\mu(A) \hat{\theta} p}\left(\frac{1}{\mu(A)}\right)^{\frac{q}{\hat{\theta} p}-1} d \nu+\int_{\theta A \backslash C} \frac{q}{\mu(A) \hat{\theta} p} d \nu \\
& \quad-\int_{\theta B \backslash C} \frac{q}{\mu(B) \hat{\theta} p} d \nu
\end{aligned}
$$

whereby

$$
\int_{\theta A \cap C} \frac{q}{\mu(A) \hat{\theta} p}\left(\frac{1}{\mu(A)}\right)^{\frac{q}{\hat{\theta}_{p}}-1} d \nu=-\int_{\theta B \cap C} \frac{q}{\mu(B) \hat{\theta} p}\left(\frac{1}{\mu(B)}\right)^{\frac{q}{\hat{\theta}_{p}}-1} d \nu
$$

This is only possible if both are zero, i.e., if $\nu(\theta A \cap C)=\nu(\theta B \cap C)=0$.

We have shown that $\nu(\theta A \cap C)=\nu(\theta B \cap C)=0$ for every $A, B \in \mathfrak{B}$ disjoint of finite non zero measure. If $N$ had dimension $\leq 1$ this would be vacuous, but as we assume that it has dimension $\geq 2$ we have in fact $\nu(\theta A \cap C)=0$ for all $A \in \mathfrak{B}$ such that $\mu(A)<\infty$. It follows that $\nu(C)=\nu\left(Y_{0} \cap C\right)=0$, i.e., that $\hat{\theta} p=q \chi_{Y_{0}}$.

Now let $A \in \mathfrak{B}$ be of finite non zero measure, $h=\mu(A)^{-1 / p(x)}$. Then $\Theta(h)=1 \Longrightarrow$ $1=\Theta^{\prime}(\theta(h))=\nu(\theta A) / \mu(A)$.

Remark A special case of this result was independently obtained at the same time by Poitevin and Raynaud [12, Lemma 6.1]. 
The technical assumption that $\theta$ sends characteristic functions to such (i.e., acts on measurable sets) is easy to obtain via a density change:

Lemma 1.9 Let $N=L_{p(\cdot)}(X, \mathfrak{B}, \mu)$ and $N^{\prime}=L_{q(\cdot)}(Y, \mathfrak{C}, \nu)$ be two Nakano spaces, and let $\theta: N \rightarrow N^{\prime}$ be an isometric embedding of Banach lattices. Then there is a measure $\lambda$ on $(Y, \mathfrak{C})$, equivalent to $\nu$, such that $D_{\nu, \lambda} \circ \theta: N \rightarrow N^{\prime \prime}=L_{q(\cdot)}(Y, \mathfrak{C}, \lambda)$ sends characteristic functions to characteristic functions, where $D_{\nu, \lambda}: N^{\prime} \rightarrow N^{\prime \prime}$ is the density change isomorphism from Fact 1.5.

Proof Let $\left\{X_{i}\right\}_{i \in I} \subseteq \mathfrak{B}$ and $\left\{Y_{j}\right\}_{j \in J} \subseteq \mathfrak{C}$ witness that $X$ and $Y$ are strictly localisable. Possibly replacing them with refinements as in Fact 1.3 we may assume that $I \subseteq J$ and that for $i \in I$ the set $Y_{i}$ is the support of $\theta\left(\chi_{X_{i}}\right)$. Define $\zeta: Y \rightarrow \mathbb{R}^{+}$by

$$
\zeta=\sum_{i \in I} \theta\left(\chi_{X_{i}}\right)^{q}+\sum_{j \in J \backslash I} \chi_{Y_{j}}
$$

This function is measurable and non zero a.e., allowing us to define another measure $\lambda$ by $d \lambda=\zeta d \nu$. Then $\nu$ and $\lambda$ are equivalent measures, and $D_{\nu, \lambda} \circ \theta\left(\chi_{X_{i}}\right)=\chi_{Y_{i}}$. Since this is an embedding of Banach lattices it follows that it sends every characteristic function to a characteristic function.

Putting everything together we obtain:

Theorem 1.10 Let $N=L_{p(\cdot)}(X, \mathfrak{B}, \mu)$ and $N^{\prime}=L_{q(\cdot)}(Y, \mathfrak{C}, \nu)$ be two Nakano spaces, $\operatorname{dim} N \geq 2$, and let $\theta: N \rightarrow N^{\prime}$ be an isometric embedding of Banach lattices. Then up to a measure density change on $Y$ :

(i) $\theta$ sends characteristic functions to such.

(ii) $\hat{\theta} p=q \chi_{\hat{\theta} X}$.

(iii) For all finite measure $A: \nu(\theta A)=\mu(A)$.

Proof Immediate from Lemma 1.9 and Lemma 1.8.

Corollary 1.11 Let $(N, \Theta)=L_{p(\cdot)}(X, \mathfrak{B}, \mu),\left(N^{\prime}, \Theta^{\prime}\right)=L_{q(\cdot)}(Y, \mathfrak{C}, \nu)$ be two Nakano spaces, $\operatorname{dim} N \geq 2$, and let $\theta: N \rightarrow N^{\prime}$ be an embedding of Banach lattices. Then $\theta$ respects the modular functional: $\Theta=\Theta^{\prime} \circ \theta$. 
Proof According to Fact 1.5 a density change on $Y$ does not alter $\Theta^{\prime}$. Thus we may assume that $\theta$ is as in the conclusion of Theorem 1.10. By Lemma 1.7 we then obtain for all $f \in N$ :

$$
\Theta^{\prime} \circ \theta(f)=\int|\theta(f)|^{q} d \nu=\int|\theta(f)|^{\hat{\theta} p} d \nu=\int \hat{\theta}\left(|f|^{p}\right) d \nu=\int|f|^{p} d \mu=\Theta(f) .
$$

Corollary 1.12 Let $(N, \Theta)=L_{p(\cdot)}(X, \mathfrak{B}, \mu)$ and $\left(N^{\prime}, \Theta^{\prime}\right)=L_{q(\cdot)}(Y, \mathfrak{C}, \nu)$ be two Nakano spaces, $\operatorname{dim} N \geq 2$, and let $\theta: N \rightarrow N^{\prime}$ be an embedding of Banach lattices. Then ess $\operatorname{rng} p \subseteq$ ess $\operatorname{rng} q$. If the band generated by $\theta(N)$ in $N^{\prime}$ is all of $N^{\prime}$ (so in particular, if $\theta$ is an isomorphism) then ess $\operatorname{rng} p=\operatorname{ess} \operatorname{rng} q$.

Proof The density change does not modify $p$ and thus neither its range, so again we may assume that $\theta$ is as in the conclusion of Theorem 1.10. It is also not difficult to see that ess $\operatorname{rng} p=\operatorname{ess} \operatorname{rng} \hat{\theta} p \backslash\{0\} \subseteq$ ess $\operatorname{rng} q$. If the band generated by $\theta(N)$ in $N^{\prime}$ is all of $N$ then $\hat{\theta} X=Y$ and $q=\hat{\theta} p$.

In the case where $\theta$ is an isomorphism this has already been proved by Poitevin [11, Proposition 3.4.4].

\section{Model theory of Nakano spaces}

\subsection{The model theoretic setting}

We assume familiarity with the general setting of continuous first order logic, as exposed in [6] or [5]. Since continuous logic only allows bounded metric structures we cannot treat Banach spaces directly. The two standard solutions for this are either to consider a Banach space as a multi-sorted structure, with a sort for $\bar{B}(0, n)$ (the closed ball of radius $n$ ) for each $n$, or to restrict our consideration to the first of these sorts, i.e., the closed unit ball. (There exists also a third solution which we shall not consider here, namely to treat the entire Banach lattice as an unbounded metric structure, see [2].) While Poitevin chose to use the former we consider the latter to be preferable, so a few words regarding the difference in approaches is in order.

The unit ball of a Banach space is, first of all, a complete convex space, i.e., a complete metric space equipped with a convex combination operation from an ambient Banach space. Such structures were characterised by Machado [9] in a language containing all 
convex combinations, and this characterisation can be expressed in continuous logic. There are advantages to a minimalistic language, though, so we prefer to work in a language consisting of a single function symbol $\frac{x+y}{2}$. Convex combinations with coefficients of the form $\frac{k}{2^{n}}$ can be obtained as more complex terms in this language, and arbitrary convex combinations with real coefficients are obtained as limits (as our structures are by definition complete), so this language is quite sufficient. While it follows from Machado's work that an axiomatisation of unit balls of Banach spaces exists in this language, it seems preferable to put an explicit axiomatisation of this kind on record along with a complete (outline of a) proof.

Let $T_{c v x}$ consist of the following axioms:

$$
\forall x\left[\frac{x+x}{2}=x\right], \quad \text { i.e., } \quad \sup _{x}\left[d\left(\frac{x+x}{2}, x\right)\right]=0,
$$

(PRM) $\forall x y z t\left[\frac{1}{2}\left(\frac{x+y}{2}+\frac{z+t}{2}\right)=\frac{1}{2}\left(\frac{z+x}{2}+\frac{t+y}{2}\right)\right]$,

etc.

(HOM) $\forall x y z\left[d\left(\frac{x+z}{2}, \frac{y+z}{2}\right)=\frac{d(x, y)}{2}\right]$.

we shall usually be interested in subsets of Banach spaces which are not only convex, but also contain zero and are symmetric around it (i.e., $-x$ exists for all $x$ ). The unit ball is such a space, but is not the only interesting one (another one is the unit ball of a von Neumann algebra with a normalised finite trace $\tau$ : it is a proper subset of the unit ball of the Hilbert spaces with inner product $\left.\langle x, y\rangle=\tau\left(x^{*} y\right)\right)$. The natural language for such symmetric convex spaces is

$$
\mathcal{L}_{B s}=\left\{0,-, \frac{x+y}{2},\|\cdot\|\right\} .
$$

we shall use $\frac{x-y}{2}$ as shorthand for $\frac{x+(-y)}{2}$. Since we wish to admit the unit ball of a Banach space as a structure in this language we shall interpret the distinguished distance symbol as half the usual distance $d(x, y)=\left\|\frac{x-y}{2}\right\|$, noticing the latter is an atomic formula. We define $T_{s c}$ (for symmetric convex) as $T_{c v x}$ along with:

(SYM)

(NORM)

$$
\begin{aligned}
& \forall x\left[\frac{x-x}{2}=0\right] \\
& \forall x\left[d(x, 0)=\frac{1}{2}\|x\|\right]
\end{aligned}
$$

Finally, we define $T_{B s}$, the theory of (unit balls of) Banach spaces as $T_{s c}$ along with

(FULL) $\forall x \exists y\left[\|x\| \geq \frac{1}{2}\right.$ or $\left.\frac{y}{2}=x\right] \quad$ i.e., $\quad \sup _{x} \inf _{y}\left[\left(\frac{1}{2}-\|x\|\right) \wedge d\left(\frac{y+0}{2}, x\right)\right]=0$

Theorem 2.1 (i) The models of $T_{c v x}$ are precisely complete convex subsets of diameter $\leq 1$ of Banach spaces. 
(ii) The models of $T_{s c}$ are precisely complete convex subsets of unit balls of Banach spaces which are symmetric around zero.

(iii) The models of $T_{B s}$ are precisely closed unit balls of Banach spaces.

Proof For each of the assertions it is clear that all the said structures are models, so we prove the converse. we shall start by examining the case of $T_{c v x}$, reducing it to that of $T_{s c}$.

From the axioms we can deduce commutativity and a variant of the triangle inequality:

$(\mathrm{COMM})$

$$
\begin{gathered}
\frac{x+y}{2}=\frac{1}{2}\left(\frac{x+x}{2}+\frac{y+y}{2}\right)=\frac{1}{2}\left(\frac{y+x}{2}+\frac{y+x}{2}\right)=\frac{y+x}{2} \\
d\left(\frac{x+y}{2}, \frac{z+w}{2}\right) \leq d\left(\frac{x+y}{2}, \frac{z+y}{2}\right)+d\left(\frac{z+y}{2}, \frac{z+w}{2}\right)=\frac{d(x, z)+d(y, w)}{2}
\end{gathered}
$$

Now let $C \vDash T_{c v x}$. Let $C-C$ be the set of all formal differences $x-y$ for $x, y \in C$, and define $d_{0}\left(x-x^{\prime}, y-y^{\prime}\right)=d\left(\frac{x+y^{\prime}}{2}, \frac{y+x^{\prime}}{2}\right)$. This is a pseudo-metric. Indeed, symmetry and reflexivity are clear, and for transitivity one checks:

$$
\begin{aligned}
d\left(\frac{x+z^{\prime}}{2}, \frac{z+x^{\prime}}{2}\right) & =2 d\left(\frac{1}{2}\left(\frac{x+z^{\prime}}{2}+\frac{y+y^{\prime}}{2}\right), \frac{1}{2}\left(\frac{z+x^{\prime}}{2}+\frac{y+y^{\prime}}{2}\right)\right) \\
& =2 d\left(\frac{1}{2}\left(\frac{x+y^{\prime}}{2}+\frac{y+z^{\prime}}{2}\right), \frac{1}{2}\left(\frac{z+y^{\prime}}{2}+\frac{y+x^{\prime}}{2}\right)\right) \\
& \leq \text { (TRI2) } d\left(\frac{x+y^{\prime}}{2}, \frac{y+x^{\prime}}{2}\right)+d\left(\frac{y+z^{\prime}}{2}, \frac{z+y^{\prime}}{2}\right)
\end{aligned}
$$

Thus $d_{0}(x-y, z-t)=0$ defines an equivalence $\sim$ relation on $C-C$, and $d_{0}$ induces a metric on $C_{-}=(C-C) / \sim=\{[x-y]: x, y \in C\}$. It is straightforward to verify that $\frac{[x-y]+[z-t]}{2}=\left[\frac{x+z}{2}-\frac{y+t}{2}\right], 0=[x-x]$ and $-[x-y]=[y-x]$ are well defined and render $C_{-}$a model of $T_{s c}$. Finally, if $x_{0} \in C$ is any fixed element then $x \mapsto\left[x-x_{0}\right]$ is an embedding of $C$ in $C_{-}$which respects convex combination and shrinks distances by a factor of 2 . It follows that if we prove that $C_{-}$embeds in a Banach space, so does $C$. We thus reduced the first assertion to the second.

We now work modulo $T_{s c}$. First, observe that $d(x, y)=2 d\left(\frac{x-y}{2}, \frac{y-y}{2}\right)=2 d\left(\frac{x-y}{2}, 0\right)=$ $\left\|\frac{x-y}{2}\right\|$. Thus the relation between the distance and the norm is as expected.

A similar reasoning shows that $\frac{x+y}{2}=0$ implies $d(y,-x)=2 d\left(\frac{x+y}{2}, \frac{x-x}{2}\right)=0$, so $y=-x$. It follows that $-(-x)=x$ and that $-\frac{x+y}{2}=\frac{-x-y}{2}$ (since $\frac{1}{2}\left(\frac{x+y}{2}+\frac{-x-y}{2}\right)=$ $\left.\frac{1}{2}\left(\frac{x-x}{2}+\frac{y-y}{2}\right)=\frac{0+0}{2}=0\right)$.

Fix a model $S \vDash T_{s c}$. For $x \in S$, let us define $\frac{1}{2} x=\frac{x+0}{2}$, and by induction we can further define $2^{-n} x$ for all $n$. If there is $y$ such that $x=\frac{1}{2} y$ then $y$ is unique (indeed, if $z$ were another such element then $0=d(x, x)=\frac{1}{2} d(y, z)$ so $y=z$ ), and we may unambiguously write $y=2 x$. If $2 \frac{x+y}{2}$ exists we write it as $x+y$. It follows from the 
definition that $x+0=x$ and $x+(-x)=0$. By definition we have $\frac{1}{2}(x+y)=\frac{x+y}{2}$ (provided that $x+y$ exists), and applying the permutation axiom we get $\frac{1}{2} x+\frac{1}{2} y=\frac{x+y}{2}$, from which it follows that $\frac{1}{2}(-x)=-\frac{1}{2} x$ and $\frac{1}{2}(x+y)=\frac{1}{2} x+\frac{1}{2} y$ (provided $x+y$ exists).

From the commutativity of $\frac{x+y}{2}$ it follows that $x+y=y+x$, by which we mean that one exists if and only if the other does, in which case they are equal. Similarly, by the permutation axioms, if $x+y$ and $y+z$ exist then $(x+y)+z=x+(y+z)$. This means we can write something like $\sum_{i<k} x_{i}$ unambiguously, without having to specify either parentheses or order, as long as we know that for every subset $w \subseteq k$ the partial sum $\sum_{i \in w} x_{i}$ exists in some order and with some organisation of the parentheses. In particular, this means that $\sum_{i<m} k_{i} 2^{-n_{i}} x_{i}$ always makes sense for $n_{i} \in \mathbb{N}, k_{i} \in \mathbb{Z}$ satisfying $\sum 2^{-n_{i}}\left|k_{i}\right| \leq 1$, and that sums and differences of such expressions behave as expected (in particular: $2^{-n-1} x+2^{-n-1} x=2^{-n} x$ ). It follows that $k 2^{-n}\left(\ell 2^{m} x\right)=$ $(k \ell) 2^{-n-m} x$.

It follows directly from the axioms that $\left\|\frac{1}{2} x\right\|=\frac{1}{2} d\left(\frac{1}{2} x, 0\right)=\frac{1}{2} \cdot \frac{1}{2} d(x, 0)=\frac{1}{2}\|x\|$. We obtain $\|x\|=2 d(0, x)=2\left\|\frac{0-x}{2}\right\|=\|-x\|$, and if $x+y$ exists then $\|x+y\|=$ $2 d(x+y, 0) \leq 2 d(x+y, y)+2 d(y, 0)=2\left\|\frac{(x+y)-y}{2}\right\|+2\left\|\frac{y-0}{2}\right\|=\|x\|+\|y\|$. By induction on $n$ one proves first that $\left\|2^{-n} x\right\|=2^{-n}\|x\|$, and then that for all $0 \leq k \leq 2^{n}$ : $\left\|k 2^{-n} x\right\|=k 2^{-n}\|x\|$. It follows that $\left\|\sum_{i<m} k_{i} 2^{-n} x_{i}\right\| \leq 2^{-n} \sum\left|k_{i}\right|$.

Thus for every $\alpha \in[-1,1]$ we can define $\alpha x$ as a limit of $k_{n} 2^{-n} x$. We obtain that $\sum \alpha_{i} x_{i}$ always makes sense if $\sum\left|\alpha_{i}\right| \leq 1, \alpha(\beta x)=(\alpha \beta) x,(\alpha+\beta) x=\alpha x+\beta x$ (provided that $|\alpha+\beta| \leq 1), \alpha(x+y)=\alpha x+\alpha y$ (provided that $x+y$ exists), and $\|\alpha x\|=|\alpha|\|x\|$. We also have $d(\alpha x, \alpha y)=\left\|\frac{\alpha x-\alpha y}{2}\right\|=|\alpha|\left\|\frac{x-y}{2}\right\|=|\alpha| d(x, y)$, so in particular $\alpha x=\alpha y \Longrightarrow x=y$ for $|\alpha| \neq 0$.

We can now define $B_{0}=\mathbb{R}^{>0} \times S$, and define $(\alpha, x) \sim(\beta, y)$ if $\frac{\alpha}{\alpha+\beta} x=\frac{\beta}{\alpha+\beta} y$. It is straightforward to verify using results from the previous paragraph that $\sim$ is an equivalence relation, and that the following operations are well defined on $B=B_{0} / \sim$ and render it a normed vector space over $\mathbb{R}$ :

$$
\begin{aligned}
& \beta[\alpha, x]= \begin{cases}{[\alpha \beta, x]} & \beta>0 \\
{[-\alpha \beta,-x]} & \beta<0 \\
{[1,0]} & \beta=0\end{cases} \\
& {[\alpha, x]+[\beta, y]=\left[\alpha+\beta, \frac{\alpha}{\alpha+\beta} x+\frac{\beta}{\alpha+\beta} y\right]} \\
& \|[\alpha, x]\|=\alpha\|x\| .
\end{aligned}
$$


Our structure $S$ embeds in the unit ball of $B$ via $x \mapsto[1, x]$.

The last assertion now follows immediately.

When dealing with models of $T_{B S}$ we allow ourselves to omit the halving operation when no ambiguity may arise. Thus, for example, we write $x+y+z=t+w$ instead of $\frac{1}{2}\left(\frac{x}{2}+\frac{y+z}{2}\right)=\frac{1}{2} \frac{t+w}{2}$, and so on.

We shall now extend this to Banach lattices. We recall a few definitions from [10]:

Definition 2.2 (i) An ordered vector space $(E, \leq)$ is a vector space $E$ equipped with a partial ordering $\leq$, over an ordered field $(k, \leq)$, satisfying

$$
v \leq u \quad \Longleftrightarrow \quad v+w \leq u+w \quad \Longleftrightarrow \quad \alpha v \leq \alpha u
$$

for all $v, u, w \in E$ and $\alpha \in k^{>0}$.

(ii) An ordered vector space is a vector lattice (or a Riesz space) if it is a lattice, i.e., if every two $v, u \in E$ admit a least upper bound (or join) $v \vee u$ and a greatest lower bound (or meet) $v \wedge u$. In this case we write $|v|=v \vee(-v), v^{+}=v \vee 0$, $v^{-}=(-v) \vee 0$.

(iii) A normed vector lattice is a vector lattice over $\mathbb{R}$, equipped with a norm satisfying $|v| \leq|u| \Longrightarrow\|v\| \leq\|u\|$.

(iv) A Banach lattice is a complete normed vector lattice.

We shall consider (unit balls of) Banach lattices in a language augmented with a 1-Lipschitz function symbol:

$$
\mathcal{L}_{B l}=\mathcal{L}_{B s} \cup\{|\cdot|\} .
$$

Using the function symbol $|\cdot|$ we may define other common expressions which have the intended interpretations in Banach lattices:

$$
\begin{array}{cl}
x^{+}=\frac{|x|+x}{2}, & x^{-}=\frac{|x|-x}{2}, \\
\frac{x \vee y}{2}=\frac{1}{2}\left(\frac{x+y}{2}+\left|\frac{x-y}{2}\right|\right), & \frac{x \wedge y}{2}=\frac{1}{2}\left(\frac{x+y}{2}-\left|\frac{x-y}{2}\right|\right) .
\end{array}
$$

On the other hand we cannot expect to define $x \vee y$ or $x \wedge y$ without halving since the unit ball of a Banach lattice need not be closed under these operations. 
We define $T_{B l}$ to consist of $T_{B s}$ along with the following axioms. We shall follow the convention (which will be justified later) that $x \geq 0$ is shorthand for $x=|x|$.

$$
\begin{array}{ll}
\text { (BL1) } & |\alpha x|=|\alpha||x| \\
\text { (BL2) } & \frac{|x|+|y|}{2} \geq 0 \\
\text { (BL3) } & \|x\|=\||x|\| \leq\||x|+|y|\| \\
\text { (BL4) } & \left|x^{+}\right|=x^{+} \\
\text {(BL5) } & \frac{z}{2}-\frac{x \vee y}{2}+\left(\frac{z-x}{2}\right)^{-}+\left(\frac{z-y}{2}\right)^{-} \geq 0
\end{array}
$$

(Some halving is omitted from BL3,5.)

Theorem 2.3 If $(E, \leq)$ is a Banach lattice then the unit ball of $E$ is closed under the absolute value operation $|\cdot|$ and as a $\mathcal{L}_{B l}$-structure is a model of $T_{B l}$. Conversely, every model of $T_{B l}$ is the unit ball of a Banach lattice, where the absolute value operation is extended to the entire Banach space by $|x|=\|x\|\left|\frac{x}{\|x\|}\right|$ and the order is recovered by $x \leq y \Longleftrightarrow x-y=|x-y|$.

Proof The first statement is immediate so we only prove the converse. If $\left(E_{1},|\cdot|\right) \vDash T_{B l}$ then $E$ is the unit ball of a Banach space $E$. By BL1 we may extend the absolute value operation to all of $E$ as in the statement of the Theorem and have $|\alpha x|=|\alpha||x|$ for all $\alpha \in \mathbb{R}, x \in E$. By BL2 ||$x|+| y||=|x|+|y|$.

Define a relation $\leq$ on $E$ as in the statement. Clearly $x-x=0=|0|$ whereby $x \leq x$. If $x \leq y \leq x$ then $0=|x-y|+|y-x|$ and by BL3 $\|x-y\| \leq 0$, i.e., $x=y$. If $x \leq y \leq z$ then $z-x=|y-x|+|z-y|$ whereby $z-x=|z-x|$, i.e., $x \leq z$. Thus $\leq$ is an ordering and it is now clear that it renders $E$ an ordered vector space. In particular, $x \geq 0 \Longleftrightarrow x=|x|$, justifying our notation.

Define $x \vee y=\frac{x+y}{2}+\left|\frac{x-y}{2}\right|, x \wedge y=\frac{x+y}{2}+\left|\frac{x-y}{2}\right|$. Then $x \vee y-x=\left(\frac{y-x}{2}\right)^{+}$, and by BL4 $x \vee y \geq x$. The inequalities $x \vee y \geq y$ and $x, y \geq x \wedge y$ are proved similarly. Assume now that $z \geq x, y$. Then $\left(\frac{z-x}{2}\right)^{-}=\frac{1}{2}(z-x)^{-}$and similarly for $z-y$, and by BL5 $z \geq x \vee y$. Thus $x \vee y$ is the join of $x$ and $y$. It is not difficult to check that $x \wedge y=-((-x) \vee(-y))$ is the meet of $x$ and $y$, so $(E, \leq)$ is a Riesz space. Immediate calculations also reveal that $|x|=x \vee(-x), x^{+}=x \vee 0, x^{-}=(-x) \vee 0$.

Finally, if $|x| \leq|y|$, applying BL3 to $|x|$ and $|y|-|x|$ we obtain $\|x\|=\||x|\| \leq\||y|\|=$ $\|y\|$. This completes the proof.

There is nothing sacred in our choice of language, and some may prefer to name the operations $\frac{x \vee y}{2}, \frac{x \wedge y}{2}$ instead of the absolute value, thus working in $\mathcal{L}_{B l}^{\prime}=\mathcal{L}_{B s} \cup$ 
$\left\{\frac{x \vee y}{2}, \frac{x \wedge y}{2}\right\}$. We have seen that $\frac{x \vee y}{2}, \frac{x \wedge y}{2}$ can be written as terms using $|\cdot|$, so every atomic $\mathcal{L}_{B l}^{\prime}$-formula can be translated to an atomic $\mathcal{L}_{B l}$-formula. The converse is not true, but we may still write $\frac{|x|}{2}=\frac{x \vee(-x)}{2}$. An easy induction on the complexity of terms yields that every atomic $\mathcal{L}_{B l}$-formula can be expressed as an atomic $\mathcal{L}_{B l}^{\prime}$-formula up to a multiplicative factor of the form $2^{k}$, and therefore as a quantifier-free $\mathcal{L}_{B l^{-}}^{\prime}$ formula. We may therefore say that the two languages are quantifier-free interpretable in one another. By Theorem A.9, model theoretic properties such as axiomatisability, quantifier elimination, model completeness, and so on, transfer from any class of Banach lattices viewed as structures in one language to the same class viewed as structures in the other. One could also formalise Banach lattices by naming the operation $x^{+}$(or $x^{-}$), and the same argument would hold.

Since we are dealing specifically with Nakano spaces, we may consider them in the language $\mathcal{L}_{B l}^{\Theta}=\mathcal{L}_{B l} \cup\{\Theta\}$ where $\Theta$ will interpret the modular functional. However, there is a small caveat here: the modular functional $\Theta_{p(\cdot)}$ is indeed uniformly continuous on the unit ball of $L_{p(\cdot)}(X, \mathfrak{B}, \mu)$, but its precise uniform continuity modulus depends on the essential bound of the exponent function $p$.

Convention 2.4 We fix here, once and for all, a uniform bound $1 \leq r<\infty$ on $p$. Thus all Nakano spaces considered henceforth will be of the form $L_{p(\cdot)}(X, \mathfrak{B}, \mu)$ where $p: X \rightarrow[1, r]$.

Let $K \subseteq[1, r]$ be compact. we shall consider the following classes of structures:

$$
\begin{aligned}
\mathcal{N}_{K}^{\Theta} & =\left\{\mathcal{L}_{B l^{-}}^{\Theta} \text { structures isomorphic to some }\left(L_{p(\cdot)}(X, \mathfrak{B}, \mu), \Theta_{p(\cdot)}\right) \text { with ess } \operatorname{rng} p=K\right\}, \\
\mathcal{N}_{K} & =\left\{\left.N\right|_{\mathcal{L}_{B l}}: N \in \mathcal{N}_{K}^{\Theta}\right\} \\
& =\left\{\mathcal{L}_{B l} \text {-structures isomorphic to some } L_{p(\cdot)}(X, \mathfrak{B}, \mu) \text { with ess } \operatorname{rng} p=K\right\}, \\
\mathcal{N}_{\subseteq K}^{\Theta} & =\bigcup\left\{\mathcal{N}_{K^{\prime}}^{\Theta}: \varnothing \neq K^{\prime} \subseteq K \text { compact }\right\}, \\
& =\left\{\mathcal{L}_{B l^{-}}^{\Theta} \text {-structures isomorphic to some }\left(L_{p(\cdot)}(X, \mathfrak{B}, \mu), \Theta_{p(\cdot)}\right) \text { with ess } \operatorname{rng} p \subseteq K\right\}, \\
\mathcal{N}_{\subseteq K} & =\bigcup\left\{\mathcal{N}_{K^{\prime}}: \varnothing \neq K^{\prime} \subseteq K \text { compact }\right\}=\left\{N \uparrow_{\left.\mathcal{L}_{B l}: N \in \mathcal{N}_{\subseteq K}^{\Theta}\right\}}\right\} \\
& =\left\{\mathcal{L}_{B l} \text {-structures isomorphic to some } L_{p(\cdot)}(X, \mathfrak{B}, \mu) \text { with ess } \operatorname{rng} p \subseteq K\right\} .
\end{aligned}
$$

(Of course, strictly speaking, these are the classes of the unit balls rather than of entire spaces.)

Given the uniform bound we fixed before, the largest classes we may consider are $\mathcal{N}_{\subseteq[1, r]}$ and $\mathcal{N}_{\subseteq[1, r]}^{\Theta}$, respectively.

Fact 2.5 Each of the classes $\mathcal{N}_{K}^{\Theta}, \mathcal{N}_{K}, \mathcal{N}_{\subseteq K}^{\Theta}$ and $\mathcal{N}_{\subseteq K}$ is elementary. 
Proof This is just [11, Proposition 3.8.2]. While the case of $\mathcal{N}_{\subseteq K}^{\Theta}$ is not mentioned there explicitly all the ingredients are there (in particular, as each class of the form $\mathcal{N}_{K}^{\Theta}$ is closed under ultraroots, so are classes of the form $\mathcal{N}_{\subseteq K}^{\Theta}$ ).

We may impose additional requirement, such as the dimension being greater than 1 , or the lattice (equivalently, the underlying measure space) being atomless. These are first order conditions as well. For the first one we would like to say that there are functions $x$ and $y$ such that $\|x\|=\|y\|=1$ and $|x| \wedge|y|=0$, i.e.:

$$
\inf _{x, y} \neg\|x\| \vee \neg\|y\| \vee\||x+y|-|x-y|\|=0 .
$$

Similarly, atomlessness is expressible by:

$$
\operatorname{supinf}_{x}\left|\|y\|-\frac{\|x\|}{2}\right| \vee \||x|-|x-2 y|||=0 .
$$

The classes of Nakano spaces of dimension at least 2 will be denoted $2 \mathcal{N}_{K}, 2 \mathcal{N}_{K}^{\Theta}$, etc. The classes of atomless Nakano spaces will be denoted $\mathcal{A N}_{K}, \mathcal{A N} \mathcal{N}_{K}^{\Theta}$, etc.

Fact 2.6 Assume $L_{p(\cdot)}(X, \mathfrak{B}, \mu) \in 2 \mathcal{N}_{K}\left(\in 2 \mathcal{N}_{\subseteq K}\right)$. Then ess $\operatorname{rng} p=K(\subseteq K)$.

Proof This is a consequence of [11, Proposition 3.4.4], which can be also obtained as a special case of Corollary 1.12.

Fact 2.7 The theory $\operatorname{Th}\left(\mathcal{A N} \mathcal{N}_{K}^{\Theta}\right)$ eliminates quantifiers. It follows that it is complete, as is $\operatorname{Th}\left(\mathcal{A N}_{K}\right)$.

Proof [11, Theorem 3.9.4].

Fact 2.8 Let $K \subseteq(1, \infty)$ be compact (so $\min K>1$ ). Then the theory $\operatorname{Th}\left(\mathcal{A} \mathcal{N}_{K}\right)$ is stable.

Proof [11, Theorem 3.10.9].

In fact, we are cheating here a little, as Poitevin proved his results in a somewhat different language. He follows the approach described in the paragraphs following [6, Example 4.5], viewing a Banach space $N$ as multi-sorted structure consisting of a sort $N_{m}=\bar{B}(0, m)$ for each $0<m<\omega$. The corresponding language for Banach lattices, which we may denote here by $\mathcal{L}_{B l, \omega}$, consists of the obvious embedding mappings between sorts, plus multiplication by (say, rational) scalars and the binary operations 
,$+ \wedge$ and $\vee$ going from sorts or pairs of sorts to an appropriate target sort (e.g., $+: N_{m} \times N_{k} \rightarrow N_{m+k}$, or $\left.\frac{1}{2} x: N_{2} \rightarrow N_{1}\right)$. The predicate symbols norm and distance can have values greater than one, but they are still bounded on each sort and thus still fit in the framework of continuous logic. Similarly, one can define $\mathcal{L}_{B l, \omega}^{\Theta}$ as $\mathcal{L}_{B l, \omega}$ along with a predicate symbol $\Theta$ on each sort, and again in every Nakano space $\Theta$ is uniformly continuous and bounded on each sort.

It will be convenient to notice that even in this approach, multiple sorts are not required. Since all the sorts $N_{m}$ of a Banach space stand in a natural bijection with the unit ball sort $N_{1}$ via dilation $x \mapsto \frac{x}{m}$, we may interpret the entire language $\mathcal{L}_{B l, \omega}$ on the single sort $N_{1}$. Thus, for example, instead of $+: N_{m} \times N_{k} \rightarrow N_{m+k}$ we would have $+_{m, k}: N_{1} \times N_{1} \rightarrow N_{1}$ sending $\left(\frac{x}{m}, \frac{y}{k}\right) \mapsto \frac{x+y}{m+k}$. Viewing $N_{1}$ as itself, rather than as a scaled copy of $N_{m}, N_{k}$ or $N_{m+k}$, obtain the convex combination operation $x+{ }_{m, k} y=\frac{m x+k y}{m+k}$. In particular, $x+{ }_{1,1} y=\frac{x+y}{2}$.

Viewed in this way, $\mathcal{L}_{B l}\left(\mathcal{L}_{B l}^{\Theta}\right)$ is a sub-language of $\mathcal{L}_{B l, \omega}\left(\mathcal{L}_{B l, \omega}^{\Theta}\right)$. It is also fairly immediate to check that every atomic $\mathcal{L}_{B l, \omega}$-formula agrees (in any Banach lattice) with a quantifier-free $\mathcal{L}_{B l}$-formula. Thus $\mathcal{L}_{B l}$ and $\mathcal{L}_{B l, \omega}$ are quantifier-free bi-interpretable, in the sense of Appendix A.2, on the class of Banach lattices. By Theorem A.9, model theoretic properties such as elementarity, model completeness, quantifier elimination, and so on, transfer between classes of Banach lattices formalised in $\mathcal{L}_{B l}$ and in $\mathcal{L}_{B l, \omega}$. (The reader may worry that in the single sorted versions of $\mathcal{L}_{B l}$ and $\mathcal{L}_{B l}^{\Theta}$ we may construct terms and formulae which do not come from the multi-sorted version due to sort discrepancy, for example the term $x+{ }_{m, k}\left(y+{ }_{\ell, t} z\right)$ where $k \neq \ell+t$. This term, however, agrees with the "legitimate" term $x+{ }_{m(\ell+t), k(\ell+t)}\left(y+{ }_{k \ell, k t} z\right)$ in every Banach lattice. In this fashion we can translate every term or quantifier-free formula of $\mathcal{L}_{B l, \omega}$ to one which would make sense in the multi-sorted version, so this is not a true problem.)

Let us now consider the case of $\mathcal{L}_{B l}^{\Theta} \subseteq \mathcal{L}_{B l, \omega}^{\Theta}$. The language $\mathcal{L}_{B l, \omega}^{\Theta}$ contains for every $m$ a predicate symbol $\Theta_{m}: N_{1} \rightarrow \mathbb{R}^{+}, \Theta_{m}(x)=\Theta(m x)$ (the range of $\Theta_{m}$ is bounded and the bound depends only on $r$ and $m$ ), while $\mathcal{L}_{B l}^{\Theta}$ only contains the first one of those, $\Theta=\Theta_{1}$. Unlike the predicates for norm and distance on $N_{m}$ which are homogeneous and can therefore be recovered from their counterparts on $N_{1}$ by simple dilation, in order to recover $\Theta_{m}$ from $\Theta_{1}$ a little more work is required. Our argument here is very close to the proof of [11, Lemma 3.4.1]. Let us first recall a version of the Stone-Weierstrass Density Theorem:

Fact 2.9 Let $X$ be a compact Hausdorff space and let $\mathscr{A} \subseteq \mathcal{C}(X, \mathbb{R})$ be a sub-algebra which separates points and vanishes nowhere (i.e., for each $x \in X$ there is $f \in \mathscr{A}$ such that $f(x) \neq 0)$. Then $\mathscr{A}$ is dense in $\mathcal{C}(X, \mathbb{R})$. 
Lemma 2.10 For every $0<m \in \mathbb{N}$ there exists a quantifier-free $\mathcal{L}_{B l}^{\Theta}$-definable predicate $\varphi_{m}(x)$ which coincides with $v \mapsto \Theta(m v)$ on the unit ball of every Nakano space $N=L_{p(\cdot)}(X, \mathfrak{B}, \mu)$ (with ess $\left.\operatorname{rng}(p) \subseteq[1, r]\right)$.

Proof Let $\mathscr{A} \subseteq \mathcal{C}([1, r], \mathbb{R})$ consist of all functions of the form $f(x)=\sum_{i<n} a_{k} 2^{-k x}$, where $n \in \mathbb{N}$ and $a_{k} \in \mathbb{R}$. Then $\mathscr{A}$ satisfies the assumptions of the Stone-Weierstrass Density Theorem cited above, and is therefore dense in $\mathcal{C}([0,1], \mathbb{R})$.

Let us fix $\varepsilon>0$. By the previous paragraph there is a function of the form $f(x)=\sum_{k<n} a_{k} 2^{-k x} \in \mathscr{A}$ which is $\varepsilon$-close to $g(x)=m^{x}$ on $[1, r]$. Then $\varphi_{m, \varepsilon}(v)=\sum_{k<n} a_{k} \Theta\left(2^{-k} v\right)$ is a quantifier-free definable predicate in $\mathcal{L}_{B l}^{\Theta}$.

Now assume that $v \in N=L_{p(\cdot)}(X, \mathfrak{B}, \mu),\|v\| \leq 1$. Passing to $|v|$ we may assume that $v \geq 0$ and up to a density change we may assume that $v=\chi_{A}$ for some $A \in \mathfrak{B}$. Then $\|v\| \leq 1$ implies that $\mu(A) \leq 1$. Consider the restriction $p \uparrow_{A}: A \rightarrow[1, r]$, and let $\nu$ be the image measure of $\mu \Gamma_{A}$ under this mapping. For every $\alpha>0$ we have

$$
\Theta(\alpha v)=\int_{A} \alpha^{p(x)} d \mu=\int_{[1, r]} \alpha^{x} d \nu
$$

whereby

$$
\begin{aligned}
\left|\Theta(m v)-\varphi_{m, \varepsilon}(v)\right| & =\left|\int_{[1, r]} m^{x} d \nu-\sum_{k<n} \int_{[1, r]} 2^{-k x} d \nu\right| \\
& \leq \int_{[1, r]}|f(x)-g(x)| d \nu \leq \varepsilon \mu(A) \leq \varepsilon .
\end{aligned}
$$

Since this can be done for every $\varepsilon>0$ the statement is proved.

Thus $\mathcal{L}_{B l}^{\Theta}$ and $\mathcal{L}_{B l, \omega}^{\Theta}$ are also quantifier-free bi-interpretable for Nakano spaces, so axiomatisability, quantifier elimination and so on transfer between the two formalisms. This also means that once we show that the modular functional of a Nakano space is $\mathcal{L}_{B l}$-definable in the unit ball (e.g., Theorem 3.1), it follows that it is $\mathcal{L}_{B l}$-definable on the $m$-ball for every $m$.

\section{Definability of the modular functional}

This section contains the main model theoretic results of this paper. We start with the definability result. 
Theorem 3.1 The modular functional $\Theta$ is uniformly $\mathcal{L}_{B l}$-definable in $2 \mathcal{N}_{\subseteq[1, r]}^{\Theta}$. Moreover, it is both uniformly inf-definable and sup-definable and can be used to axiomatise $2 \mathcal{N}_{\subseteq[1, r]}^{\Theta}$ modulo the axioms for $2 \mathcal{N}_{\subseteq[1, r]}$.

More precisely:

(i) There exists a $\mathcal{L}_{B l}$-definable predicate $\varphi_{\Theta}(x)$ such that $(N, \Theta) \vDash \Theta(x)=\varphi_{\Theta}(x)$ for all $(N, \Theta) \in 2 \mathcal{N}_{\subseteq[1, r]}^{\Theta}$.

(ii) There quantifier-free $\mathcal{L}_{B l}$-formulae $\psi_{n}\left(x, \bar{y}_{n}\right)$ and $\chi_{n}\left(x, \bar{z}_{n}\right)$ such that in all Nakano spaces of dimension at least two:

$$
\Theta(x)=\varphi_{\Theta}(x)=\lim _{n \rightarrow \infty} \inf _{\bar{y}_{n}} \psi_{n}\left(x, \bar{y}_{n}\right)=\lim _{n \rightarrow \infty} \sup _{\bar{z}_{n}} \chi_{n}\left(x, \bar{z}_{n}\right),
$$

each of the limits converging uniformly and at a uniform rate.

(iii) The theory $\operatorname{Th}\left(2 \mathcal{N}_{\subseteq[1, r]}^{\Theta}\right)$ is equivalent to $\operatorname{Th}\left(2 \mathcal{N}_{\subseteq[1, r]}\right) \cup\left\{\Theta(x)=\varphi_{\Theta}(x)\right\}$.

Proof By Corollary 1.11 every $N \in 2 \mathcal{N}_{\subseteq[1, r]}$ admits at most one expansion to $(N, \Theta) \in 2 \mathcal{N}_{\subseteq[1, r]}^{\Theta}$. As these are elementary classes, one can apply Theorem A.1 (Beth's theorem for continuous logic) in order to obtain $\varphi_{\Theta}$.

Using Corollary 1.11 again we see that $\varphi_{\Theta}$ is constant in $2 N_{\subseteq[1, r]}$ (see Definition A.2). By Theorem A.4 it is both inf-definable and sup-definable there.

The last item is immediate.

Corollary 3.2 For a fixed compact $K \subseteq[1, r]$, the modular functional is uniformly $\mathcal{L}_{B l}$-definable in $\mathcal{N}_{K}^{\Theta}$.

In particular the modular functional is $\mathcal{L}_{B l}$-definable in every Nakano Banach lattice.

Proof If $K=\left\{p_{0}\right\}$ is a single point, we have $\Theta(f)=\|f\|^{p_{0}}$. Otherwise $\mathcal{N}_{K}^{\Theta}=$ $2 \mathcal{N}_{K}^{\Theta} \subseteq 2 \mathcal{N}_{\subseteq[1, r]}^{\Theta}$ and we can apply Theorem 3.1.

We have shown that naming the modular functional does not add structure. Still, in the case of an atomless Nakano space naming $\Theta$ does give something, namely quantifier elimination. It is clear that without $\Theta$ quantifier elimination would be impossible: the complete $\mathcal{L}_{B l}^{\Theta}$-type of a function contains, among other information, the essential range of $p$ on its support, and there is no way of recovering this information from the quantifier-free $\mathcal{L}_{B l}$-type of a single positive function, as it is determined by its norm alone.

A next-best would be to obtain model completeness. Indeed, all the work for obtaining it is already done. 
Theorem 3.3 For every compact $K \subseteq[1, r]$ the (theory of the) class $\mathcal{A N}_{K}$ is model complete.

Proof Follows from Corollary 1.11 and the quantifier elimination in $\mathcal{A} \mathcal{N}_{K}^{\Theta} \cdot \quad \mathbf{\square}_{3.3}$

The next and last result of this section is quite quick and straightforward to prove for a person who is quite familiar with the notion of a measure algebra and understands that Theorem 1.10 is actually a result about measure algebras rather than about measure spaces. Having intentionally avoided all mention of measure algebras so far, a longer approach is required, presenting this somewhat different point of view. We introduce measure algebras in a very sketchy fashion, as an abstract version of a (strictly localisable) measure space. For a comprehensive treatment we refer the reader to [8].

Let $(X, \mathfrak{B}, \mu)$ be a measure space. Let $\mathfrak{B}_{f} \subseteq \mathfrak{B}$ be the lattice of finite measure sets. As an algebraic structure, $\left(\mathfrak{B}_{f}, \cup, \cap, \backslash\right)$ is a relatively complemented distributive lattice (and if it contains a maximal element then it is a Boolean algebra). The measure $\mu: \mathfrak{B}_{f} \rightarrow \mathbb{R}^{+}$induces a pseudo-metric $d(x, y)=\mu(x \triangle y)$ on $\mathfrak{B}_{f}$. The kernel of this pseudo-metric, namely the equivalence relation $d(x, y)=0$, is compatible with the algebraic structure, yielding a quotient relatively complemented distributive lattice $(\overline{\mathfrak{B}}, \cap, \cup, \backslash)$. The measure function $\mu$ induces an additive "measure function" $\bar{\mu}: \overline{\mathfrak{B}} \rightarrow \mathbb{R}^{+}$, and $d(x, y)=\bar{\mu}(x \triangle y)$ is a metric on $\overline{\mathfrak{B}}$, with respect to which the operations $\cap, \cup, \backslash$ are 1-Lipschitz. Moreover, it follows from $\sigma$-additivity of the original measure that $\overline{\mathfrak{B}}$ is a complete metric space. For the purpose of the discussion that follows, we call $(\overline{\mathfrak{B}}, \cup, \cap, \backslash, \bar{\mu})$ the measure algebra associated to $(X, \mathfrak{B}, \mu)$.

Conversely, let $(\mathfrak{C}, \cup, \cap, \backslash, \nu)$ be an abstract measure algebra, namely a relatively complemented distributive lattice where $\nu: \mathfrak{C} \rightarrow \mathbb{R}^{+}$is additive, such that in addition $d(x, y)=\nu(x \triangle y)$ is a complete metric on $\mathfrak{C}$. Assume first that $\mathfrak{C}$ contains a maximal element 1, i.e., that $\mathfrak{C}$ is a Boolean algebra. Let $\widetilde{\mathfrak{C}}$ be its Stone space. For $x \in \mathfrak{C}$ let $\tilde{x} \subseteq \widetilde{\mathfrak{C}}$ be the corresponding clopen set, and define $\tilde{\nu}_{0}(\tilde{x})=\nu(x)$. Then Carathéodory's Extension Theorem applies and we may extend $\tilde{\nu}_{0}$ uniquely to a regular Borel measure $\tilde{\nu}$ on $\widetilde{\mathfrak{C}}$. It is now easy to check that $(\mathfrak{C}, \cup, \cap, \backslash, \nu)$ is the measure algebra associated to the measure space $(\widetilde{\mathfrak{C}}, \tilde{\nu})$ (equipped with the Borel $\sigma$-algebra). In the general case let $\left\{a_{i}\right\}_{i \in I} \subseteq \mathfrak{C}$ be a maximal disjoint family of non-zero members. For each $i$ let $\mathfrak{C}_{i}=\left\{b \cap a_{i}\right\}_{b \in \mathfrak{C}}$ be the restriction of $\mathfrak{C}$ to $a_{i}$. Restriction the other operations we obtain a measure algebra $\left(\mathfrak{C}_{i}, \cup, \cap, \backslash, \nu_{i}\right)$ with a maximal element $a_{i}$, so the previous argument works. The disjoint union $\coprod_{i \in I}\left(\widetilde{\mathfrak{C}}_{i}, \tilde{\nu}_{i}\right)$ is a strictly localisable measure space, and it is not difficult to check that its measure algebra is (canonically identified with) $\mathfrak{C}$. 
Definition 3.4 Let $\alpha>0$ be an ordinal, $\left\{N_{i}\right\}_{i<\alpha}$ an increasing chain of members of $2 \mathcal{N}_{\subseteq[1, r]}$ (as usual, all inclusions are assumed to be isometric).

A compatible presentation for this sequence is a sequence of presentations $N_{i} \cong$ $L_{p_{i}(\cdot)}\left(X_{i}, \mathfrak{B}_{i}, \mu_{i}\right)$ such that each inclusion $N_{i} \subseteq N_{j}$ sends characteristic functions to characteristic functions.

Lemma 3.5 Let $\alpha>0$ be a limit ordinal, $\left\{N_{i}\right\}_{i<\alpha}$ an increasing chain of members of $2 \mathcal{N}_{\subseteq[1, r]}$. Let $N_{i} \cong L_{p_{i}(\cdot)}\left(X_{i}, \mathfrak{B}_{i}, \mu_{i}\right), i<\alpha$, be a compatible presentation for this sequence. Let $N_{\alpha}=\bigcup_{i<\alpha} N_{i}$ in the sense of continuous logic, namely the metric completion of the set-theoretic union. Then $N_{\alpha} \in 2 \mathcal{N}_{\subseteq[1, r]}$ as well.

Moreover, there exists a presentation $N_{\alpha} \cong L_{p_{\alpha}(\cdot)}\left(X_{i}, \mathfrak{B}_{i}, \mu_{i}\right)$ which extends the original compatible presentation to one for the sequence $\left\{N_{i}\right\}_{i \leq \alpha}$, and ess $\operatorname{rng} p_{\alpha}=$ $\overline{\bigcup_{i} \text { ess rng } p_{i}}$

Proof For $i<\alpha$ let $\overline{\mathfrak{B}}_{i}$ be the measure algebra associated to the measure space $\left(X_{i}, \mathfrak{B}_{i}, \mu_{i}\right)$. The compatibility assumption tells us precisely that for $i<j<\alpha$, the embedding $N_{i} \subseteq N_{j}$ induces an embedding $\overline{\mathfrak{B}}_{i} \subseteq \overline{\mathfrak{B}}_{j}$ which respects the algebraic structure as well as the measure. We may therefore define $\mathfrak{C}={\widehat{U_{i<\alpha} \overline{\mathfrak{B}}_{i}}}_{\text {(i.e., the }}$ completion of the union). Since the algebraic (lattice) operations as well as the measure function are uniformly continuous, they extend uniquely to $\mathfrak{C}$, rendering it an abstract measure algebra. By the discussion above we may identify it with the measure algebra $\overline{\mathfrak{B}}_{\alpha}$ of some measure space $\left(X_{\alpha}, \mathfrak{B}_{\alpha}, \mu_{\alpha}\right)$.

For each $i<j \leq \alpha$, the embedding $\overline{\mathfrak{B}}_{i} \subseteq \overline{\mathfrak{B}}_{j}$ induces a partial mapping $L_{0}\left(X_{i}, \mathfrak{B}_{i}, \mu_{i}\right) \rightarrow L_{0}\left(X_{j}, \mathfrak{B}_{j}, \mu_{j}\right)$ defined on the space of simple functions. By Lemma 1.6 this extends uniquely to a total mapping $\hat{\theta}_{i j}: L_{0}\left(X_{i}, \mathfrak{B}_{i}, \mu_{i}\right) \rightarrow$ $L_{0}\left(X_{j}, \mathfrak{B}_{j}, \mu_{j}\right)$. Moreover, for $i<j<k \leq \alpha$ we have $\hat{\theta}_{j k} \circ \hat{\theta}_{i j}=\hat{\theta}_{i k}$ and $\left.\hat{\theta}_{i j}\right|_{N_{i}}$ coincides with the inclusion $N_{i} \subseteq N_{j}$.

By Theorem 1.10 we have $\hat{\theta}_{i j} p_{i}=\chi_{\hat{\theta}_{i j} X_{i}} p_{j}$, whence $\left.\hat{\theta}_{i \alpha} p_{i}\right|_{\hat{\theta}_{i \alpha} X_{i}}=\hat{\theta}_{j \alpha} p_{j} \uparrow_{\hat{\theta}_{i \alpha} X_{i}}$ for $i<j<\alpha$. It is also not difficult to check that $X_{\alpha}=\bigcup_{i<\alpha} \hat{\theta}_{i \alpha} X_{i}$ up to null measure (more precisely, that every finite measure $A \in \mathfrak{B}_{\alpha}$ is contained, up to arbitrarily small measure, by some $\hat{\theta}_{i \alpha} X_{i}$ ), so there exists a unique measurable $p_{\alpha}: X_{\alpha} \rightarrow[1, r]$ such that $\hat{\theta}_{i \alpha} p_{i}=\chi_{\hat{\theta}_{i \alpha} X_{i}} p_{\alpha}$ for all $i<\alpha$, and its essential range is as stated. Let $N_{\alpha}^{\prime}=L_{p_{\alpha}(\cdot)}\left(X_{\alpha}, \mathfrak{B}_{\alpha}, \mu_{\alpha}\right)$. We obtain embeddings $\hat{\theta}_{i, \alpha} \uparrow_{N_{i}}: N_{i} \rightarrow N_{\alpha}^{\prime}$. Moreover, every characteristic function of a finite measure set in $N_{\alpha}^{\prime}$ is arbitrarily well approximated by members of the set union $\bigcup_{i} \hat{\theta}_{i, \alpha}\left(N_{i}\right)$ (by construction of $\mathfrak{C}$ ). It follows that the image of the set union is dense, whence we get an isomorphism $N_{\alpha} \cong N_{\alpha}^{\prime}=L_{p_{\alpha}(\cdot)}\left(X_{\alpha}, \mathfrak{B}_{\alpha}, \mu_{\alpha}\right)$ which respects characteristic functions, as desired. 
Theorem 3.6 The (theories of the) classes $\mathcal{N}_{K}, \mathcal{N}_{\subseteq K}, \mathcal{N}_{K}^{\Theta}, \mathcal{N}_{\subseteq K}^{\Theta}$, and similarly with prefixes 2 and $\mathcal{A}$, are all inductive.

Proof It is immediate from the previous Lemma that $2 \mathcal{N}_{\subseteq K}$ and $2 \mathcal{N}_{\subseteq K}^{\Theta}$ are inductive. It follows that $\mathcal{N}_{\subseteq K}$ and $\mathcal{N}_{\subseteq K}^{\Theta}$ are inductive, since every infinite increasing chain in this classes has a tail in $2 \mathcal{N}_{\subseteq K}^{\subseteq}$ or in $2 \mathcal{N}_{\subseteq K}^{\Theta}$. Since the atomlessness axiom is inductive, the classes $\mathcal{A} \mathcal{N}_{\subseteq K}$ and $\mathcal{A} \mathcal{N}_{\subseteq K}^{\Theta}$ are inductive. The same reasoning works for $K$ instead of $\subseteq K$. (Of course, for $\mathcal{A} \mathcal{N}_{K}$ and $\mathcal{A} \mathcal{N}_{K}^{\Theta}$, inductiveness follows directly from model completeness).

\section{Perturbations of the exponent}

Intuitively, a small change to the exponent function $p$ should not change the structure of a Nakano space by too much. We formalise this intuitive idea, showing that small perturbations of the exponent form indeed a perturbation system in the sense of [2]. We show that up to such perturbations, every complete theory of Nakano spaces is $\aleph_{0}$-categorical and $\aleph_{0}$-stable. In case $p$ is constant (i.e., $K$ is a singleton), we already know (see, e.g., [4]) that the theory is $\aleph_{0}$-stable and $\aleph_{0}$-categorical without perturbation. Indeed, no perturbation of $p$ is possible in this case, so it is a special case of what we prove below.

\subsection{Preliminary computations}

We seek bounds for $1+\gamma^{s}$ in terms of $(1+\gamma)^{s}$, and for $1-\gamma^{s}$ in terms of $(1-\gamma)^{s}$, where $\gamma \in[0,1]$ and $s \in[1 / r, r]$. The function $\frac{1+\gamma^{s}}{(1+\gamma)^{s}}$ is well behaved, i.e., continuous as a function of two variables, and will not cause trouble. The function $\frac{1-\gamma^{s}}{(1-\gamma)^{s}}$ is badly behaved near $\gamma=1$, so we shall only use it for $\gamma \in\left[0, \frac{1}{2}\right]$. For $\gamma \in\left[\frac{1}{2}, 1\right]$ we shall have to consider another function, namely $\varphi(\gamma, s)=\frac{\ln \left(1-\gamma^{s}\right)}{\ln (1-\gamma)}$, which is, for the time being, only defined for $\gamma \in(0,1)$ and $s>0$. We calculate its limit as $\gamma \rightarrow 1$ for a fixed $s>0$ making several uses of l'Hôpital's rule (marked with $*$ ):

$$
\begin{aligned}
\lim _{\gamma \rightarrow 1} \frac{\ln \left(1-\gamma^{s}\right)}{\ln (1-\gamma)} & ={ }^{*} \lim _{\gamma \rightarrow 1} \frac{-s \gamma^{s-1}\left(1-\gamma^{s}\right)^{-1}}{-(1-\gamma)^{-1}}=\lim _{\gamma \rightarrow 1} \frac{s \gamma^{s-1}(1-\gamma)}{1-\gamma^{s}} \\
& ={ }^{*} \lim _{\gamma \rightarrow 1} \frac{s(s-1) \gamma^{s-2}-s^{2} \gamma^{s-1}}{-s \gamma^{s-1}}=\frac{-s}{-s}=1 .
\end{aligned}
$$

It is therefore natural to extend $\varphi$ by $\varphi(1, s)=1$. This function is continuous in each variable for $s>0$ and $\gamma \in(0,1]$, and we wish to show that it is continuous 
as a function of two variables. In fact, all we need is to show it is continuous on $\left[\frac{1}{2}, 1\right] \times[1, r]$.

Assume $\gamma \in(0,1), s \in[1, r]$. A straightforward verification leads to:

$$
\frac{\ln \left(1-\gamma^{r}\right)}{\ln (1-\gamma)} \leq \frac{\ln \left(1-\gamma^{s}\right)}{\ln (1-\gamma)} \leq 1
$$

whereby:

$$
\left|1-\frac{\ln \left(1-\gamma^{s}\right)}{\ln (1-\gamma)}\right| \leq\left|1-\frac{\ln \left(1-\gamma^{r}\right)}{\ln (1-\gamma)}\right|
$$

Thus $\lim _{\gamma \rightarrow 1} \frac{\ln \left(1-\gamma^{s}\right)}{\ln (1-\gamma)}=1$ uniformly for $s \in[1, r]$, and $\varphi(\gamma, s)$ is indeed continuous on $\left[\frac{1}{2}, 1\right] \times[1, r]$.

We now define for $1 \leq s \leq r$ :

$$
\begin{aligned}
& A_{s}=\inf \left\{\frac{\ln \left(1-\gamma^{s}\right)}{s \ln (1-\gamma)}: \gamma \in\left[\frac{1}{2}, 1\right)\right\} \leq \frac{1}{s}, \\
& B_{s}^{-}=\sup \left\{\frac{1-\gamma^{t}}{(1-\gamma)^{t}}: \gamma \in\left[0, \frac{1}{2}\right], t \in[1 / s, s]\right\}, \\
& B_{s}^{+}=\sup \left\{\frac{1+\gamma^{t}}{(1+\gamma)^{t}}: \gamma \in[0,1], t \in[1 / s, s]\right\}, \\
& B_{s}=\max \left\{B_{s}^{-}, B_{s}^{+}\right\} .
\end{aligned}
$$

By continuity of $\varphi(\gamma, s)$, and since $\varphi(\gamma, 1)=1$ for all $\gamma: \lim _{s \rightarrow 1} A_{s}=\lim _{s \rightarrow 1} \frac{1}{s}=1$. Similarly $\lim _{s \rightarrow 1} B_{s}=1$.

In particular we have for $s \in[1, r]$ and $\gamma \in\left[\frac{1}{2}, 1\right): A_{s} \leq \frac{\ln \left(1-\gamma^{s}\right)}{s \ln (1-\gamma)}$ whereby $s A_{s} \ln (1-$ $\gamma) \geq \ln \left(1-\gamma^{s}\right)$ and thus $(1-\gamma)^{s A_{s}} \geq 1-\gamma^{s}$.

Lemma 4.1 Let $\alpha, \beta \in[-1,1]$ and $1 / s \leq t \leq s$. Then

$$
\left.|\operatorname{sgn}(\alpha)| \alpha\right|^{t}-\operatorname{sgn}(\beta)|\beta|^{t} \mid \leq \max \left\{|\alpha-\beta|^{A_{s} t}, B_{s}|\alpha-\beta|^{t}\right\} .
$$

Proof We may assume that $|\alpha| \geq|\beta|$ by symmetry. We may further assume that $\alpha, \beta \neq 0$. Assume first that $\operatorname{sgn}(\alpha \beta)=-1$. Then:

$$
\begin{aligned}
\left.|\operatorname{sgn}(\alpha)| \alpha\right|^{t}-\operatorname{sgn}(\beta)|\beta|^{t} \mid & =|\alpha|^{t}\left(1+|\beta / \alpha|^{t}\right) \\
& \leq|\alpha|^{t} B_{s}(1+|\beta / \alpha|)^{t} \\
& =B_{s}|\alpha-\beta|^{t} .
\end{aligned}
$$


A similar argument shows that when $\operatorname{sgn}(\alpha \beta)=1$ and $|\beta / \alpha| \leq 1 / 2$ :

$$
\left.|\operatorname{sgn}(\alpha)| \alpha\right|^{t}-\operatorname{sgn}(\beta)|\beta|^{t}\left|\leq B_{s}\right| \alpha-\left.\beta\right|^{t} .
$$

Finally, assume $\operatorname{sgn}(\alpha \beta)=1$ and $|\beta / \alpha| \geq 1 / 2$. We use the fact that $|\alpha| \leq 1$ and $A_{s} \leq 1 / s<1$ imply that $|\alpha| \leq|\alpha|^{A_{s}}$ :

$$
\begin{aligned}
\left.|\operatorname{sgn}(\alpha)| \alpha\right|^{t}-\operatorname{sgn}(\beta)|\beta|^{t} \mid & =|\alpha|^{t}\left(1-|\beta / \alpha|^{t}\right) \leq|\alpha|^{t}\left(1-|\beta / \alpha|^{s}\right) \\
& \leq|\alpha|^{t}(1-|\beta / \alpha|)^{A_{s} s} \\
& \leq|\alpha|^{A_{s} t}(1-|\beta / \alpha|)^{A_{s} t} \\
& =|\alpha-\beta|^{A_{s} t} .
\end{aligned}
$$

This completes the proof.

Lemma 4.2 For all $\gamma, t \in[0,1]: t(1-\gamma)+\gamma^{t} \leq 1$ (where $\left.0^{0}=1\right)$.

Proof This is clear for $t \in\{0,1\}$. So let $t \in(0,1)$, and let $f_{t}(\gamma)=t(1-\gamma)+\gamma^{t}$. Then $f_{t}(1)=1$, and for $0<\gamma<1$ and $t-1<0$ we have $\gamma^{t-1}>1$ whereby:

$$
\frac{d}{d \gamma} f_{t}=-t+t \gamma^{t-1}>-t+t=0 \text {. }
$$

Thus $f_{t}(\gamma) \leq 1$ for all $\gamma \in[0,1]$.

For $1 \leq s \leq r$ and $0 \leq x \leq 2$, define:

$$
\begin{gathered}
\eta_{s}(x)= \begin{cases}x^{A_{s} / s} & x \leq 1 \\
x^{s} & 1<x \leq 2\end{cases} \\
\hat{\eta}_{s}(x)=2^{1-A_{s}} B_{s} \eta_{s}(x) / A_{s} .
\end{gathered}
$$

Lemma 4.3 As $s \rightarrow 1$, the functions $\eta_{s}$ converge uniformly to the identity. As a consequence, $\hat{\eta}_{s} \rightarrow$ id uniformly as $s \rightarrow 1$.

Proof For $\eta_{s}$, one verifies uniform convergence separately for $x \mapsto x^{A_{s} / s}$ on $[0,1]$ and for $x \mapsto x^{s}$ on $[1,2]$. Uniform convergence of $\hat{\eta}_{s}$ follows.

For $1 \leq s \leq r$, define:

$$
\begin{aligned}
& C_{s}^{1}=\sup \left\{\left.\left|\operatorname{sgn}\left(\frac{\alpha+\beta}{2}\right)\right| \frac{\alpha+\beta}{2}\right|^{t}-\frac{\operatorname{sgn}(\alpha)|\alpha|^{t}+\operatorname{sgn}(\beta)|\beta|^{t}}{2} \mid: \alpha, \beta \in[-1,1], t \in[1 / s, s]\right\}, \\
& C_{s}^{2}=\sup \left\{\left|x-\hat{\eta}_{s}(x)\right|: x \in[0,2]\right\}, \\
& C_{s}=\max \left\{C_{s}^{1}, C_{s}^{2}\right\} .
\end{aligned}
$$

Then $\lim _{s \rightarrow 1} C_{s}=0$. 


\subsection{Perturbing the exponent}

Definition 4.4 Let $(X, \mathfrak{B}, \mu)$ be a measure space and $p, q: X \rightarrow[1, r]$ measurable. We define $\mathscr{E}_{p, q}: L_{0}(X, \mathfrak{B}, \mu) \rightarrow L_{0}(X, \mathfrak{B}, \mu)$ by:

$$
\left(\mathscr{E}_{p, q} f\right)(x)=\operatorname{sgn}(f(x))|f(x)|^{p(x) / q(x)} .
$$

Lemma 4.5 We continue with the assumptions of Definition 4.4. Let $(N, \Theta)=$ $\left(L_{p(\cdot)}(X, \mathfrak{B}, \mu), \Theta_{p(\cdot)}\right)$ and $\left(N^{\prime}, \Theta^{\prime}\right)=\left(L_{q(\cdot)}(X, \mathfrak{B}, \mu), \Theta_{q(\cdot)}\right)$.

(i) For each $f \in L_{0}(X, \mathfrak{B}, \mu)$ we have $\Theta(f)=\Theta^{\prime}\left(\mathscr{E}_{p, q} f\right)$. Thus in particular $\mathscr{E}_{p, q}$ sends $N$ into $N^{\prime}$ and the unit ball of $N$ into the unit ball of $N^{\prime}$.

(ii) The mapping $\mathscr{E}_{p, q}$ is bijective, its inverse being $\mathscr{E}_{q, p}$. It restricts to a bijection between $N$ and $N^{\prime}$, as well as to a bijection between their respective unit balls.

(iii) The mapping $\mathscr{E}_{p, q}$ commutes with measure density change. More precisely, assume $\nu$ is another measure on $(X, \mathfrak{B})$, equivalent to $\mu$, say $d \nu(x)=\zeta(x) d \mu(x)$. Let $M=L_{p(\cdot)}(X, \mathfrak{B}, \nu), M^{\prime}=L_{q(\cdot)}(X, \mathfrak{B}, \nu)$. Let $D_{\mu, \nu}^{p}: N \rightarrow M$ and $D_{\mu, \nu}^{q}: N^{\prime} \rightarrow M^{\prime}$ be the respective density change mappings. Then $D_{\mu, \nu}^{q} \circ \mathscr{E}_{p, q}=$ $\mathscr{E}_{p, q} \circ D_{\mu, \nu}^{p}: N \rightarrow M^{\prime}$.

Proof For the first item we calculate that:

$$
\Theta^{\prime}\left(\mathscr{E}_{p, q} f\right)=\int|f(x)|^{p(x)} d \mu=\Theta(f) .
$$

The second item follows. Finally, we calculate:

$$
\begin{aligned}
\left(D_{\mu, \nu}^{q} \mathscr{E}_{p, q} f\right)(x) & =\zeta(x)^{-1 / q(x)}\left(\mathscr{E}_{p, q} f\right)(x) \\
& =\zeta(x)^{-1 / q(x)} \operatorname{sgn}(f(x))|f(x)|^{p(x) / q(x)} \\
& =\operatorname{sgn}\left(\zeta(x)^{-1 / p(x)} f(x)\right)\left|\zeta(x)^{-1 / p(x)} f(x)\right|^{p(x) / q(x)} \\
& =\operatorname{sgn}\left(\left(D_{\mu, \nu}^{p} f\right)(x)\right)\left|\left(D_{\mu, \nu}^{p} f\right)(x)\right|^{p(x) / q(x)} \\
& =\left(\mathscr{E}_{p, q} D_{\mu, \nu}^{p} f\right)(x),
\end{aligned}
$$

proving the third item.

Proposition 4.6 We continue with the notation and assumptions of Lemma 4.5. Assume that $s$ is such that $1 / s \leq q(x) / p(x) \leq s$ (for example, we can take $s=r$ ). Then for every $f, g \in N_{1}$ (the unit ball of $N$ ): $\left\|\mathscr{E}_{p, q} f-\mathscr{E}_{p, q} g\right\| \leq \hat{\eta}_{s}(\|f-g\|)$ and $\|f-g\| \leq \hat{\eta}_{s}\left(\left\|\mathscr{E}_{p, q} f-\mathscr{E}_{p, q} g\right\|\right)$. 
Proof Let $f, g \in N_{1}$. By Lemma 4.5(iii) we may assume that $|f| \vee|g|=\chi_{S}$ for some set $S \in \mathfrak{B}$, so $f(x), g(x) \in[-1,1]$. Let

$$
\begin{aligned}
h(x) & =\left.|\operatorname{sgn}(f(x))| f(x)\right|^{p(x) / q(x)}-\operatorname{sgn}(g(x))|g(x)|^{p(x) / q(x)} \mid \\
S_{1} & =\left\{x \in S: h(x) \leq|f(x)-g(x)|^{A_{s} p(x) / q(x)}\right\} \\
S_{2} & =S \backslash S_{1} \subseteq\left\{x \in S: h(x) \leq B_{S}|f(x)-g(x)|^{p(x) / q(x)}\right\} .
\end{aligned}
$$

We observe that as $\|f\|,\|g\| \leq 1$ we have $\mu(S) \leq 2$. Observe also that $A_{s} q(x) / s \leq$ $q(x) / s \leq p(x)$ and that $s q(x) / A_{s} \geq s q(x) \geq p(x)$. It follows that if $\|f-g\| \leq 1$ then:

$$
\begin{aligned}
\|f-g\|^{p(x)} & \leq \eta_{s}(\|f-g\|)^{q(x) / A_{s}}=\|f-g\|^{q(x) / s} \\
& \leq \eta_{s}(\|f-g\|)^{q(x)}=\|f-g\|^{A_{s} q(x) / s} .
\end{aligned}
$$

Otherwise $1<\|f-g\| \leq 2$, and:

$$
\begin{aligned}
\|f-g\|^{p(x)} & \leq \eta_{s}(\|f-g\|)^{q(x)}=\|f-g\|^{s q(x)} \\
& \leq \eta_{s}(\|f-g\|)^{q(x) / A_{s}}=\|f-g\|^{s q(x) / A_{s}} .
\end{aligned}
$$

Let $\gamma=\int_{S_{1}} \frac{|f(x)-g(x)|^{p(x)}}{\|f-g\| \|^{p(x)}} d \mu(x)$ and $a=\hat{\eta}_{s}(\|f-g\|)=2^{1-A_{s}} B_{s} \eta_{s}(\|f-g\|) / A_{s}$. Then:

$$
\begin{aligned}
\Theta^{\prime}\left(\frac{\mathscr{E}_{p, q} f-\mathscr{E}_{p, q} g}{a}\right) & =\int_{S} \frac{h(x)^{q(x)}}{a^{q(x)}} d \mu(x) \\
& \leq \int_{S_{1}} \frac{|f(x)-g(x)|^{A_{s} p(x)}}{a^{q(x)}} d \mu(x)+\int_{S_{2}} \frac{B_{s}^{q(x)}|f(x)-g(x)|^{p(x)}}{a^{q(x)}} d \mu(x)
\end{aligned}
$$

We work on each integral separately.

$$
\begin{aligned}
\int_{S_{1}} \frac{|f(x)-g(x)|^{A_{s} p(x)}}{a^{q(x)}} d \mu(x) & =\int_{S_{1}} \frac{\mu\left(S_{1}\right) A_{s}^{q(x)}}{\left(2^{1-A_{s}} B_{s}\right)^{q(x)}}\left(\frac{|f(x)-g(x)|^{p(x)}}{\eta_{s}(\|f-g\|)^{q(x) / A_{s}}}\right)^{A_{s}} \frac{d \mu(x)}{\mu\left(S_{1}\right)} \\
& \leq \frac{\mu\left(S_{1}\right)}{2^{1-A_{s}}} \int_{S_{1}}\left(\frac{|f(x)-g(x)|^{p(x)}}{\|f-g\|^{p(x)}}\right)^{A_{s}} \frac{d \mu(x)}{\mu\left(S_{1}\right)} \\
& \leq \frac{\mu\left(S_{1}\right)}{2^{1-A_{s}}}\left(\int_{S_{1}} \frac{|f(x)-g(x)|^{p(x)}}{\| f-g(x)} \frac{d \|^{p(x)}}{\mu\left(S_{1}\right)}\right)^{A_{s}} \\
& =\frac{\mu(S)^{1-A_{s}}}{2^{1-A_{s}}} \gamma^{A_{s}} \leq \gamma^{A_{s}}
\end{aligned}
$$

And:

$$
\begin{aligned}
\int_{S_{2}} \frac{B_{s}^{q(x)}|f(x)-g(x)|^{p(x)}}{a^{q(x)}} d \mu(x) & =\int_{S_{2}} \frac{\left(A_{s} B_{s}\right)^{q(x)}}{\left(2^{1-A_{s}} B_{s}\right)^{q(x)}} \frac{|f(x)-g(x)|^{p(x)}}{\eta_{s}\left(\left.\|f-g\|\right|^{q(x)}\right.} d \mu(x) \\
& \leq A_{s} \int_{S_{2}} \frac{|f(x)-g(x)|^{p(x)}}{\|f-g\|^{p(x)}} d \mu(x)=A_{s}(1-\gamma) .
\end{aligned}
$$


Thus:

$$
\Theta^{\prime}\left(\frac{\mathscr{E}_{p, q} f-\mathscr{E}_{p, q} g}{a}\right) \leq \gamma^{A_{s}}+A_{s}(1-\gamma) \leq 1
$$

We conclude that $\left\|\mathscr{E}_{p, q} f-\mathscr{E}_{p, q} g\right\| \leq a=\hat{\eta}_{s}(\|f-g\|)$. Since $1 / s \leq p(x) / q(x) \leq s$ as well we have $\|f-g\|=\left\|\mathscr{E}_{q, p} \mathscr{E}_{p, q} f-\mathscr{E}_{q, p} \mathscr{E}_{p, q} g\right\| \leq \hat{\eta}_{s}\left(\left\|\mathscr{E}_{p, q} f-\mathscr{E}_{p, q} g\right\|\right)$.

Corollary 4.7 The mapping $\mathscr{E}_{p, q}: N_{1} \rightarrow N_{1}^{\prime}$ is uniformly continuous, the modulus of uniform continuity depending solely on $r$.

Proof Define $\Delta_{r}(\varepsilon)=\min \left\{\left(2^{A_{r}-1} A_{r} \varepsilon / B_{r}\right)^{r / A_{r}}, 1\right\}$. Then for all $\varepsilon>0$ we have $\Delta_{r}(\varepsilon)>0$ and $\|f-g\|<\Delta_{r}(\varepsilon) \Longrightarrow\left\|\mathscr{E}_{p, q} f-\mathscr{E}_{p, q} g\right\| \leq \varepsilon$.

Proposition 4.8 Let $\mathscr{E}_{p, q}: N \rightarrow N^{\prime}$ be as in Definition 4.4, and let $f, g \in N_{1}$. Then:

(i) $\mathscr{E}_{p, q} 0=0 ;-\mathscr{E}_{p, q} f=\mathscr{E}_{p, q}(-f) ; \mathscr{E}_{p, q}(|f|)=\left|\mathscr{E}_{p, q} f\right|$

(ii) $\left|\|f-g\|-\left\|\mathscr{E}_{p, q} f-\mathscr{E}_{p, q} g\right\|\right| \leq C_{s}$.

(iii) $\left\|\mathscr{E}_{p, q} \frac{f+g}{2}-\frac{\mathscr{E}_{p, q f} f \mathscr{E}_{p, q g}}{2}\right\| \leq 2 C_{s}$.

Proof The first item is clear. For the second we use Proposition 4.6:

$$
\begin{aligned}
\left\|\mathscr{E}_{p, q} f-\mathscr{E}_{p, q} g\right\|-\|f-g\| & \leq \hat{\eta}_{s}(\|f-g\|)-\|f-g\| \leq C_{s}, \\
\|f-g\|-\left\|\mathscr{E}_{p, q} f-\mathscr{E}_{p, q} g\right\| & \leq \hat{\eta}_{s}\left(\left\|\mathscr{E}_{p, q} f-\mathscr{E}_{p, q} g\right\|\right)-\left\|\mathscr{E}_{p, q} f-\mathscr{E}_{p, q} g\right\| \leq C_{s} .
\end{aligned}
$$

We may assume that $|f| \vee|g|=\chi_{S}$ for some measurable set $S$, so $\mu(S) \leq 2$. By definition of $C_{s}$ we have $\left|\mathscr{E}_{p, q} \frac{f+g}{2}(x)-\frac{\mathscr{E}_{p, q} f+\mathscr{E}_{p, q} g}{2}(x)\right| \leq C_{s}$ for $x \in S$, and we get:

$$
\Theta\left(\frac{\mathscr{E}_{p, q} \frac{f+g}{2}-\frac{\mathscr{E}_{p, q} f+\mathscr{E}_{p, q} g}{2}}{2 C_{S}}\right) \leq \int_{S} 2^{-q(x)} d \mu(x) \leq \frac{\mu(S)}{2} \leq 1 .
$$

The third item follows.

We now wish to define a perturbation system $\mathfrak{p}$ for $\mathcal{L}_{B l}$-structures. We do this by defining a $\mathfrak{p}(\varepsilon)$-perturbation of structures $N$ and $N^{\prime}$ directly as a bijection $\theta: N \rightarrow N^{\prime}$ such that for all $f, g, h \in N$ :

$$
\begin{gathered}
\theta 0=0, \\
\theta(-f)=-\theta f, \\
\theta(|f|)=|\theta f|, \\
\left|d\left(\frac{f+g}{2}, h\right)-d\left(\frac{\theta f+\theta g}{2}, \theta h\right)\right| \leq \varepsilon, \\
|\|f\|-\|\theta f\|| \leq \varepsilon,
\end{gathered}
$$


and:

$$
e^{-\varepsilon e^{\varepsilon}} d(f, g)^{e^{\varepsilon}} \leq d(\theta f, \theta g) \leq e^{\varepsilon} d(f, g)^{e^{-\varepsilon}} .
$$

(While for most symbols we can just allow to "change by $\varepsilon$ ", we need to take special care with the distance symbol.) This indeed defines a perturbation system, as it clearly verifies the following characterisation:

Fact 4.9 Let $T$ be a theory, and assume that for each $r \in \mathbb{R}^{+}$and $M, N \in \operatorname{Mod}(T)$, $\operatorname{Pert}_{r}^{\prime}(M, N)$ is a set of bijections of $M$ with $N$ satisfying the following properties:

(i) Monotonicity: $\operatorname{Pert}_{r}^{\prime}(M, N)=\bigcap_{s>r} \operatorname{Pert}_{s}^{\prime}(M, N)$.

(ii) Non-degenerate reflexivity: $\operatorname{Pert}_{0}^{\prime}(M, N)$ is the set of isomorphisms of $M$ with $N$.

(iii) Symmetry: $f \in \operatorname{Pert}_{r}^{\prime}(M, N)$ if and only $f^{-1} \in \operatorname{Pert}_{r}^{\prime}(N, M)$.

(iv) Transitivity: if $f \in \operatorname{Pert}_{r}^{\prime}(M, N)$ and $g \in \operatorname{Pert}_{s}^{\prime}(N, L)$ then $g \circ f \in \operatorname{Pert}_{r+s}^{\prime}(M, L)$.

(v) Uniform continuity: for each $r \in \mathbb{R}^{+}$, all members of $\operatorname{Pert}_{r}^{\prime}(M, N)$, where $M, N$ vary over all models of $T$, satisfy a common modulus of uniform continuity.

(vi) Ultraproducts: If $f_{i} \in \operatorname{Pert}_{r}^{\prime}\left(M_{i}, N_{i}\right)$ for $i \in I$, and $\mathscr{U}$ is an ultrafilter on $I$ then $\prod_{\mathscr{U}} f_{i} \in \operatorname{Pert}_{r}^{\prime}\left(\prod_{\mathscr{U}} M_{i}, \prod_{\mathscr{U}} N_{i}\right)$. (Note that $\prod_{\mathscr{U}} f_{i}$ exists by the uniform continuity assumption).

(vii) Elementary substructures: If $f \in \operatorname{Pert}_{r}^{\prime}(M, N), M_{0} \preceq M$, and $N_{0}=f\left(M_{0}\right) \preceq N$ then $f \uparrow_{M_{0}} \in \operatorname{Pert}_{r}^{\prime}\left(M_{0}, N_{0}\right)$.

Then there exists a unique perturbation system $\mathfrak{p}$ for $T$ such that $\operatorname{Pert}_{r}^{\prime}(M, N)=$ $\operatorname{Pert}_{\mathfrak{p}(r)}(M, N)$ for all $r, M$ and $N$.

Proof [3, Theorem 4.4].

Recall that given two $n$-types $p, q$ we say that $d_{\mathfrak{p}}(p, q) \leq \varepsilon$ if there are $\mathcal{L}_{B l}$-structures $N, N^{\prime}$ and an $\varepsilon$-perturbation $\theta: N \rightarrow N^{\prime}$ sending a realisation of $p$ to one of $q$.

Lemma 4.10 For every $\varepsilon>0$ there exists $s>1$ such that if $N=L_{p(\cdot)}(X, \mathfrak{B}, \mu)$, $N^{\prime}=L_{q(\cdot)}(X, \mathfrak{B}, \mu)$ and $\mathscr{E}_{p, q}: N \rightarrow N^{\prime}$ is as in Definition 4.4 (so in particular $1 / s \leq$ $p(x) / q(x) \leq s$ for almost all $x \in X)$, then $\mathscr{E}_{p, q}$ is a $\mathfrak{p}(\varepsilon)$-perturbation.

Proof By Proposition 4.6, Proposition 4.8 and the fact that $\lim _{s \rightarrow 1} C_{s}=0$. 
Lemma 4.11 Fix a compact $K \subseteq[1, r]$ and $s>1$. Then there is a finite set $K_{s} \subseteq[0,1]$ such that for every atomless measure space $(X, \mathfrak{B}, \mu)$ and $p: X \rightarrow[1, r]$ with ess $\operatorname{rng}(p)=K$ there exists $q: X \rightarrow[1, r]$ such that ess $\operatorname{rng}(q)=K_{s}$ and for almost all $x \in X: 1 \leq q(x) / p(x) \leq s$.

Proof By compactness we can cover $K$ with finitely many open intervals $[1, r] \subseteq$ $\bigcup\left\{\left(a_{i}, b_{i}\right): i<n\right\}$, with $1<b_{i} / a_{i} \leq s$. We may assume that $K \cap\left(a_{i}, b_{i}\right) \neq \varnothing$ for all $i<n$. We then define $K_{s}=\left\{b_{i}: i<n\right\}$.

Assume now that $(X, \mathfrak{B}, \mu)$ is atomless and $p: X \rightarrow[1, r]$ satisfies ess $\operatorname{rng}(p)=K$. We can then split $X$ into a finite disjoint union of positive measure sets $X=\bigcup_{i<n} X_{i}$ such that the essential range of $p_{i}=p \Upsilon_{X_{i}}$ is contained in $\left(a_{i}, b_{i}\right)$. Define $q(x)=b_{i}$ when $x \in X_{i}$. Then $q$ is as required.

Fact 4.12 For $K$ consisting of a single point, the theory $\operatorname{Th}\left(\mathcal{A N} \mathcal{N}_{K}\right)$ is $\aleph_{0}$-categorical and $\aleph_{0}$-stable.

Proof [4].

Lemma 4.13 Let $K \subseteq[1, r]$ be finite. Then $\operatorname{Th}\left(\mathcal{A N} \mathcal{N}_{K}\right)$ is $\aleph_{0}$-categorical and $\aleph_{0}$ stable.

Proof Let $K=\left\{p_{i}: i<n\right\}, p_{0}<\ldots<p_{n-1}$. If $N=L_{p(\cdot)}(X, \mathfrak{B}, \mu) \in \mathcal{A N}_{K}$ then $X$ can be written as a disjoint union $X=\bigcup_{i<n} X_{i}$ where $X_{i} \in \mathfrak{B}, \mu\left(X_{i}\right)>0$ and $p\left\lceil_{X_{i}} \equiv p_{i}\right.$ a.e. For $i<n$ let $N_{i}$ be the Banach lattice $\chi_{X_{i}} N$. Thus the $N_{i}$ are orthogonal bands in $N$ and $N=\bigoplus_{i<n} N_{i}$. Since we can recover $\Theta$ from the norm on each $N_{i}$ we can recover $\Theta$ on $N$, and thus we can recover the norm on $N$. Similarly, as the $N_{i}$ are orthogonal bands we can recover the lattice structure on $N$ from that of $N_{i}$.

Now, if $N$ is separable (and atomless), each $N_{i}$ is separable and atomless, and thus uniquely determined by $p_{i}$ up to isomorphism, whereby $N$ is uniquely determined by $K$. This proves $\aleph_{0}$-categoricity.

Similarly, let $N^{\prime} \preceq N$ be a separable elementary sub-model and let $N_{i}^{\prime}=N^{\prime} \cap N_{i}$. By $\aleph_{0}$-stability of $\operatorname{Th}\left(N_{i}\right), \mathrm{S}_{\ell}^{N_{i}}\left(N_{i}^{\prime}\right)$ is metrically separable for each $i$. Now let $\bar{f}=$ $f^{0}, \ldots, f^{\ell-1} \in N$, and let $f^{j}=\sum_{i<n} f_{k}^{j}$ where $f_{i}^{j} \in N_{i}$. Naming $\Theta$ and using quantifier elimination we see that $\operatorname{tp}^{N}\left(\bar{f} / N^{\prime}\right)$ is uniquely determined by $\left(\operatorname{tp}^{N_{i}}\left(\bar{f}_{i} / N_{i}^{\prime}\right): i<n\right)$, and we might as well write $\operatorname{tp}^{N}\left(\bar{f} / N^{\prime}\right)=\sum_{i<n} \operatorname{tp}^{N_{i}}\left(\bar{f}_{i} / N_{i}^{\prime}\right)$. If $q=\sum_{i<n} q_{i}$ and $q^{\prime}=\sum_{i<n} q_{i}^{\prime}$ are two such decompositions then we have $d\left(q, q^{\prime}\right) \leq \sum_{i<n} d\left(q_{i}, q_{i}^{\prime}\right)$. Thus $\mathrm{S}_{\ell}^{N}\left(N^{\prime}\right)$ is metrically separable. 
We can now conclude:

Theorem 4.14 The theory $\operatorname{Th}\left(\mathcal{A N}_{\subseteq[1, r]}\right)$ is $\mathfrak{p}-\aleph_{0}$-stable, and every completion thereof (which is of the form $\operatorname{Th}\left(\mathcal{A N} \mathcal{N}_{K}\right)$ ) is $\mathfrak{p}$ - $\aleph_{0}$-categorical.

Proof Combining Lemma 4.10 and Lemma 4.11 we see that for every $\varepsilon>0$ there is a finite set $K^{\prime} \subseteq[1, r]$ such that every separable $N, N^{\prime} \in \mathcal{A N}_{K}$ admit $\mathfrak{p}(\varepsilon / 2)$ perturbations with separable $\tilde{N}, \tilde{N} \in \mathcal{A N}_{K^{\prime}}$, respectively. But $\tilde{N} \cong \tilde{N}^{\prime}$ by Lemma 4.13, so $N$ and $N^{\prime}$ admit a $\mathfrak{p}(\varepsilon)$-perturbation.

Similarly for $\mathfrak{p}-\aleph_{0}$-stability.

Corollary 4.15 The theory $\operatorname{Th}\left(\mathcal{A} \mathcal{N}_{\subseteq[1, r]}\right)$ is stable.

Proof By [3, Proposition 4.11] $\lambda$-p-stability implies stability. (See [3, Section 4.3] for more properties and characterisations of $\aleph_{0}$-stability up to perturbation.)

Remark It is in fact also true that the theory $\operatorname{Th}\left(\mathcal{N}_{\left\{p_{0}\right\}}\right)$ (i.e., constant $p$, but possibly with atoms) is $\aleph_{0}$-stable, although this fact is not proved anywhere in the literature at the time of writing. By the same reasoning, the theory $\operatorname{Th}\left(\mathcal{N}_{\subseteq[1, r]}\right)$ is $\mathfrak{p}$ - $\aleph_{0}$-stable and in particular stable.

\section{A Some basic continuous model theory}

\section{A.1 Definability and monotonicity}

Theorem A.1 (Beth's definability theorem for continuous logic) Let $\mathcal{L}_{0} \subseteq \mathcal{L}$ be continuous signatures with the same sorts (i.e., $\mathcal{L}$ does not add new sorts on top of those existing in $\mathcal{L}_{0}$ ) and $T$ an $\mathcal{L}$-theory such that every $\mathcal{L}_{0}$-structure $M_{0}$ admits at most a single expansion to an $\mathcal{L}$-structure $M$ which is a model of $T$. Then every symbol in $\mathcal{L}$ admits an explicit $\mathcal{L}_{0}$-definition in $T$. That is to say that for every predicate symbol $P(\bar{x}) \in \mathcal{L}$ is equal in all models of $T$ to some $\mathcal{L}_{0}$-definable predicate $\varphi_{P}(\bar{x})$, and for every function symbol $f(\bar{x}) \in \mathcal{L}$ the predicate $d(f(\bar{x}), y)$ is equal in all models of $T$ to some $\mathcal{L}_{0}$-definable predicate $\varphi_{f}(\bar{x})$.

Proof For convenience we shall assume that the language is single sorted, but the same proof holds for a many sorted language. 
Let $P \in \mathcal{L}$ be an $n$-ary function symbol, and consider the mapping $\theta_{n}: \mathrm{S}_{n}(T) \rightarrow$ $\mathrm{S}_{n}\left(\mathcal{L}_{0}\right)$, the latter being the space of all complete $n$-types in the language $\mathcal{L}_{0}$. It is known that $\theta_{n}$ is continuous, and we claim it is injective.

Indeed, let $p, p^{\prime} \in \mathrm{S}_{n}(T)$ be such that $\theta_{n}(p)=\theta_{n}\left(p^{\prime}\right)=q$. Let $M \vDash p(\bar{a})$ and $M^{\prime} \vDash p^{\prime}\left(\bar{a}^{\prime}\right)$, so Then $\operatorname{tp}^{\mathcal{L}_{0}}(\bar{a})=\operatorname{tp}^{\mathcal{L}_{0}}\left(\bar{a}^{\prime}\right)=q$.

Claim There exists an elementary extension $M \preceq M_{1}$ and an $\mathcal{L}_{0}$-elementary embed$\operatorname{ding} M^{\prime} \hookrightarrow M_{1}$ sending $\bar{a}^{\prime}$ to $\bar{a}$.

Proof of claim We need to verify that $\operatorname{Th}_{\mathcal{L}(M)}(M) \cup \operatorname{Th}_{\mathcal{L}_{0}}\left(M^{\prime}\right) \cup\left\{\bar{a}=\bar{a}^{\prime}\right\}$ is consistent. But the assumptions on the types tell us precisely that $\operatorname{Th}_{\mathcal{L}_{0}}\left(M^{\prime}\right) \cup\left\{\bar{a}=\bar{a}^{\prime}\right\}$ is approximately finitely satisfiable in $(M, \bar{a})$.

we shall identify $M^{\prime}$ as a set with its image in $M_{1}$, and in particular assume that $\bar{a}=\bar{a}^{\prime}$.

Claim Let $N$ and $N^{\prime}$ be two $\mathcal{L}$-structures, and assume that $N \preceq \mathcal{L}_{0} N^{\prime}$ (but needn't even be an $\mathcal{L}$-substructure). Then there exists $N^{\prime \prime} \succeq N$ such that $N^{\prime} \preceq \mathcal{L}_{0} N^{\prime \prime}$.

Proof of claim The assumption $N \preceq \mathcal{L}_{0} N^{\prime}$ implies that $\operatorname{Th}_{\mathcal{L}_{0}\left(N^{\prime}\right)}\left(N^{\prime}\right)$ is approximately finitely satisfiable in $N$, so $\operatorname{Th}_{\mathcal{L}(N)}(N) \cup \operatorname{Th}_{\mathcal{L}_{0}\left(N^{\prime}\right)}\left(N^{\prime}\right)$ is consistent.

Using the claim we can extend the pair $M^{\prime}=M_{0}^{\prime} \preceq \mathcal{L}_{0} M_{1}$ to a chain of $\mathcal{L}$-structures We now construct a sequence of structures $M_{0}^{\prime} \preceq \mathcal{L}_{0} M_{1} \preceq \mathcal{L}_{0} M_{1}^{\prime} \preceq \mathcal{L}_{0} M_{2} \preceq \mathcal{L}_{0} M_{2}^{\prime} \ldots$ such that $M_{i} \preceq M_{i+1}$ and $M_{i}^{\prime} \preceq M_{i+1}^{\prime}$.

Let $M_{\omega}=\bigcup M_{i}, M_{\omega}^{\prime}=\bigcup M_{i}^{\prime}$. Then both $M_{\omega}$ and $M_{\omega}^{\prime}$ are models of $T$ and have the same $\mathcal{L}_{0}$-reduct, and are therefore the same. It follows that $p=\operatorname{tp}^{M_{\omega}}(\bar{a})=\operatorname{tp}^{M_{\omega}^{\prime}}(\bar{a})=$ $p^{\prime}$.

Once we have established that $\theta_{n}$ is an injective continuous mapping between compact Hausdorff spaces it is necessarily an embedding (i.e., a homeomorphism with its image). We may identify the predicate $P$ with a continuous function $P: \mathrm{S}_{n}(T) \rightarrow[0,1]$. By Tietze's extension theorem there exists a continuous function $\varphi_{P}: \mathrm{S}_{n}\left(\mathcal{L}_{0}\right) \rightarrow[0,1]$ such that $P=\varphi_{P} \circ \theta_{n}$. Then $\varphi_{P}$ is the required $\mathcal{L}_{0}$-definable predicate.

If $f$ is a function symbol, apply the preceding argument to $d(f(\bar{x}), y)$.

$\mathbf{a}_{\text {A.1 }}$

Definition A.2 Let $T$ be a theory, $\varphi(\bar{x})$ a definable predicate. We say that $\varphi$ is increasing (decreasing) in $T$ if whenever $M \subseteq N$ are both models of $T$ and $\bar{a} \in M$ we have $\varphi(\bar{a})^{M} \leq \varphi(\bar{a})^{N}\left(\varphi(\bar{a})^{M} \geq \varphi(\bar{a})^{N}\right)$. We say that $\varphi$ is constant in $T$ if it is both increasing and decreasing in $T$. 
Definition A.3 A sup-formula is a formula of the form $\sup _{\bar{y}} \varphi(\bar{x}, \bar{y})$ where $\varphi$ is quantifier-free.

A sup-definable predicate is a definable predicate which can be written syntactically as $\mathcal{F} \lim \varphi_{n}(\bar{x})$ where each $\varphi_{n}$ is a sup-formula. (See [6, Definition 3.6] and subsequent discussion for the definition and properties of the forced limit operation $\mathcal{F}$ lim.) Notice that every such predicate is equal to a uniform limit of sup-formulae.

We make the analogous definitions for inf.

Theorem A.4 Let $T$ be a theory, $\varphi(\bar{x})$ a definable predicate. Then $\varphi$ is increasing (decreasing) in $T$ if and only if $\varphi$ is equivalent modulo $T$ to a sup-definable (inf definable) predicate.

Proof Clearly it suffices to prove the case of increasing definable predicates. Right to left being immediate, we prove left to right.

Assume therefore that $\varphi(\bar{x})$ is increasing in $T$. Let $\Psi$ be the collection of all supformulae $\psi(\bar{x})=\sup _{\bar{y}} \tilde{\psi}(\bar{x}, \bar{y})$ such that $T \vdash \psi(\bar{x}) \leq \varphi(\bar{x})$. Notice that the latter means that $T \vdash \tilde{\psi}(\bar{x}, \bar{y}) \leq \varphi(\bar{x})$. If for every $n<\omega$ there is $\psi_{n} \in \Psi$ such that $T \vdash \varphi(\bar{x})-2^{-n} \leq \psi(\bar{x})$ then $\varphi=\mathcal{F} \lim \psi_{n}$ and we are done. In order to conclude we shall assume the converse and obtain a contradiction.

We assume then that there is $n<\omega$ such that $T \cup\left\{\varphi(\bar{x})-\psi(\bar{x}) \geq 2^{-n}\right\}$ is consistent for all $\psi \in \Psi$. As $\Psi$ is closed under $\vee$ and $\varphi \cdot\left(\psi \vee \psi^{\prime}\right) \geq 2^{-n} \Longrightarrow \varphi \div \psi \geq 2^{-n}$, the set $\Sigma=T \cup\left\{\varphi \cdot \psi \geq 2^{-n}\right\}_{\psi \in \Psi}$ is consistent. Let $(M, \bar{a})$ be a model for it, and let $r=\varphi(\bar{a})^{M}$.

Let $\Sigma^{\prime}=T \cup \operatorname{Diag}_{a}(M) \cup\left\{\varphi(\bar{a}) \leq r-2^{-n}\right\}$. Here $\operatorname{Diag}_{a}(M)$ denotes the atomic diagram of $M$, namely the family of all conditions of the form $\chi(\bar{a})=\chi(\bar{a})^{M}$ where $\chi(\bar{x})$ is an atomic formula and $\bar{a} \in M$, so a model of $\operatorname{Diag}_{a}(M)$ is a structure in which $M$ is embedded. If $\Sigma^{\prime}$ were consistent we would get a contradiction to $\varphi$ being increasing, so $\Sigma^{\prime}$ is contradictory. By compactness there exists a quantifier-free formula $\chi(\bar{x}, \bar{y})$ and $\bar{b} \in M$ such that $\chi(\bar{a}, \bar{b})^{M}=0$ and $T \cup\{\chi(\bar{x}, \bar{y})=0\} \cup\left\{\varphi(\bar{x}) \leq r-2^{-n}\right\}$ is contradictory. It follows there is some $m$ such that $T \cup\left\{\chi(\bar{x}, \bar{y}) \leq 2^{-m}\right\} \cup\{\varphi(\bar{x}) \leq$ $\left.r-2^{-n}\right\}$ is contradictory. Let $r^{\prime} \in\left(r-2^{-n}, r\right)$ be a dyadic number, and let $\tilde{\psi}=r^{\prime}-2^{m} \chi$. Then $\tilde{\psi}$ is a quantifier-free formula, and we claim that $T \vdash \tilde{\psi}(\bar{x}, \bar{y}) \leq \varphi(\bar{x})$. indeed, for any model $N \vDash T$ and any $\bar{c}, \bar{d} \in N$ :

$$
\begin{aligned}
& \varphi(\bar{c})^{N} \geq r^{\prime} \quad \Longrightarrow \quad \varphi(\bar{c})^{N} \geq r^{\prime} \geq \tilde{\psi}(\bar{c}, \bar{d})^{N} \\
& \varphi(\bar{c})^{N} \leq r^{\prime} \quad \Longrightarrow \quad \chi(\bar{c}, \bar{d})^{N} \geq 2^{-m} \quad \Longrightarrow \quad \varphi(\bar{c})^{N} \geq 0=\tilde{\psi}(\bar{c}, \bar{d})^{N} .
\end{aligned}
$$


Thus $\psi(\bar{x})=\sup _{\bar{y}} \tilde{\psi}(\bar{x}, \bar{y}) \in \Psi$, whereby $\varphi(\bar{a})^{M}-\psi(\bar{a})^{M} \geq 2^{-n}$. But $\chi(\bar{a}, \bar{b})^{M}=0$, so $\psi(\bar{a}) \geq r^{\prime}$ whereby $\varphi(\bar{a})^{M} \geq r^{\prime}+2^{-n}>r$, a contradiction. This concludes the proof.

Corollary A.5 A continuous theory $T$ is model complete if and only if every formula (definable predicate) is equivalent modulo $T$ to an inf-definable predicate.

Proof Left to right is by Theorem A.4. For right to left, every formula $\varphi$ is decreasing in $T$, and considering $\neg \varphi$ every formula is increasing as well, and therefore constant in $T$, which means precisely that $T$ is model complete.

A.5

\section{A.2 Interpretations}

We turn to treat the issue of passage from one language to another in a structure, which has arisen several times in this paper. We start with a somewhat watered down notion of a structure being interpretable in another.

Definition A.6 (Interpretation schemes) Let $\mathcal{L}_{0}$ and $\mathcal{L}_{1}$ be two single sorted signatures. A (restricted) interpretation scheme $\Phi: \mathcal{L}_{0} \rightarrow \mathcal{L}_{1}$ consists of a mapping assigning to every atomic $\mathcal{L}_{1}$-formula $\varphi(\bar{x})$ an $\mathcal{L}_{0}$-definable predicate $\varphi^{\Phi}(\bar{x})$.

Let $M$ be an $\mathcal{L}_{0}$-structure. We define $\Phi(M)$ to be any $\mathcal{L}_{1}$-structure, should one exist, equipped with a mapping $\iota: M \rightarrow \Phi(M)$ with a dense image, such that for every every atomic $\mathcal{L}_{1}$-formula $\varphi(\bar{x})$ :

$$
\varphi(\iota \bar{a})^{\Phi(M)}=\varphi^{\Phi}(\bar{a})^{M} \quad \text { for all } \bar{a} \in M .
$$

It is not difficult to check that the pair $(\Phi(M), \iota)$, if it exists, is unique up to a unique isomorphism, justifying the notation. By a convenient abuse of notation we shall omit $\iota$ altogether, identifying $\bar{a} \in M$ with $\iota \bar{a} \in \Phi(M)$.

We define $\mathcal{K}^{\Phi}$ to be the class of $\mathcal{L}_{0}$-structures $M$ for which $\Phi(M)$ exists. More generally, if $\mathcal{K}$ is a class of $\mathcal{L}_{1}$-structures, we define $\Phi^{-1}(\mathcal{K})=\left\{M \in \mathcal{K}^{\Phi}: \Phi(M) \in\right.$ $\mathcal{K}\}$.

By induction on the structure of $\mathcal{L}_{1}$-formulae on extends the mapping $\varphi \mapsto \varphi^{\Phi}$ from atomic formulae to arbitrary ones. If $\varphi$ is an $\mathcal{L}_{1}$-definable predicate it can always be written as $\mathcal{F} \lim \varphi_{n}$ where $\varphi_{n}$ are formulae, and we may then define $\varphi^{\Phi}=\mathcal{F} \lim \left(\varphi_{n}\right)^{\Phi}$. It is straightforward to check that if $M \in \mathcal{K}^{\Phi}$ then (1) holds for every formula or definable predicate $\varphi$. 
We qualified this notion of interpretation as "restricted", since it uses the entire home sort of the interpreting structure, whereas the tradition notion of interpretation in classical logic allows the interpretation to take place on an arbitrary definable set. We could extend the definition by letting the domain of the mapping $\iota$, rather than be all of $M$, be some definable subset $X \subseteq M^{n}$, where $d(\bar{x}, X)$ is given uniformly by a definable predicate $\chi^{\Phi}(\bar{x})$ which is also prescribed by $\Phi$. Everything we prove here regarding interpretations goes through with this more general definition. In particular, the class of structures in which $\chi^{\Phi}$ defines the distance to a set (the zero set of $\chi^{\Phi}$ ) is elementary. For details on definable sets in continuous logic and their properties we refer the reader to [1, Section 1].

Lemma A.7 Let $\Phi: \mathcal{L}_{0} \rightarrow \mathcal{L}_{1}$ be an interpretation scheme. Then the class $\mathcal{K}^{\Phi}$ is elementary and we may write $T^{\Phi}=\operatorname{Th}\left(\mathcal{K}^{\Phi}\right)$. More generally, if $\mathcal{K}=\operatorname{Mod}(T)$ is a an elementary class of $\mathcal{L}_{1}$-structures then $\Phi^{-1}(\mathcal{K})$ is elementary as well, and we may write $\Phi^{-1}(T)=\operatorname{Th}\left(\Phi^{-1}(\mathcal{K})\right)$.

Proof In the case where $\mathcal{L}_{1}$ is purely relational, $T^{\Phi}$ merely consists of axioms expressing that the predicate symbols respect the uniform continuity moduli prescribed by $\mathcal{L}_{1}$. In case there are also function symbols we need more axioms (all free variables are quantified universally):

- Axioms expressing that $d(f(\bar{x}), y)^{\Phi}$ respects the uniform continuity moduli of $f$ in the $\bar{x}$ and is 1 -Lipschitz in $y$.

- The axioms $d(y, z)^{\Phi} \leq d(f(\bar{x}), y)^{\Phi}+d(f(\bar{x}), z)^{\Phi}$ and $\inf _{y} d(f(\bar{x}), y)^{\Phi}=0$. Notice that if $d\left(f(\bar{x}), y_{n}\right)^{\Phi} \rightarrow 0$ as $n \rightarrow \infty$ then $\left\{y_{n}\right\}$ is a Cauchy sequence and therefore admits a limit. Thus for all $\bar{x}$ there exists a unique $y$ such that $d(f(\bar{x}), y)^{\Phi}=0$, and for all other $z$ : $d(f(\bar{x}), z)^{\Phi}=d(y, z)^{\Phi}$. We may then legitimately write $y=f^{\Phi}(\bar{x})$.

- Finally, axioms expressing that other atomic formulae are interpreted appropriately. For example, for an atomic formula $P(f(x, g(y)), z)$ we need to say that $P^{\Phi}\left(f^{\Phi}\left(x, g^{\Phi}(y)\right), z\right)=P(f(x, g(y)), z)^{\Phi}$, expressed by

$$
\inf _{t, w}\left(d(g(y), w)^{\Phi} \vee d(f(x, w), t)^{\Phi} \vee\left|P(t, z)^{\Phi}-P(f(x, g(y)), z)^{\Phi}\right|\right)=0 .
$$

It is relatively straightforward to check that the collection of these axioms does define the class $\mathcal{K}^{\Phi}$.

Assume now that $\mathcal{K}=\operatorname{Mod}(T)$ is an elementary class of $\mathcal{L}_{1}$-structures and let $\Phi^{-1}(T)=T^{\Phi} \cup\left\{\varphi^{\Phi}\right\}_{\varphi \in T}$. Then $\Phi^{-1}(\mathcal{K})=\operatorname{Mod}\left(\Phi^{-1}(T)\right)$, as desired. 
Definition A.8 (Composition of interpretation schemes, bi-interpretability) Assume now that $\Psi: \mathcal{L}_{1} \rightarrow \mathcal{L}_{2}$ is another interpretation scheme. We then define an interpretation scheme $\Psi \circ \Phi: \mathcal{L}_{0} \rightarrow \mathcal{L}_{2}$ by $\varphi^{\Psi \circ \Phi}=\left(\varphi^{\Psi}\right)^{\Phi}$ for each atomic $\mathcal{L}_{2}$-formula $\varphi$. Again it is straightforward to check that $\Phi^{-1}\left(\mathcal{K}^{\Psi}\right) \subseteq \mathcal{K}^{\Psi \circ \Phi}$, and that if $M \in \Phi^{-1}\left(\mathcal{K}^{\Psi}\right)$ then $\Psi(\Phi(M))=\Psi \circ \Phi(M)$.

Finally, consider interpretation schemes $\Phi: \mathcal{L}_{0} \rightarrow \mathcal{L}_{1}$ and $\Psi: \mathcal{L}_{1} \rightarrow \mathcal{L}_{0}$, and a class $\mathcal{K}$ of $\mathcal{L}_{0}$-structures. Assume that $\mathcal{K} \subseteq \Phi^{-1}\left(\mathcal{K}^{\Psi}\right)$ and that $\Psi \circ \Phi(M)=M$ (with $\iota=\operatorname{id}_{M}$ ) for all $M \in \mathcal{K}$. We then say that $\mathcal{K}$ and $\mathcal{K}^{\prime}=\Phi(\mathcal{K})=\{\Phi(M)\}_{M \in \mathcal{K}}$ are (strongly) bi-interpretable by $(\Phi, \Psi)$. Notice that this is a symmetric notion, namely that in this case $\mathcal{K}^{\prime} \subseteq \Psi^{-1}\left(\mathcal{K}^{\Phi}\right), \mathcal{K}=\Psi\left(\mathcal{K}^{\prime}\right)$ and $\Phi \circ \Psi(N)=N$ for all $N \in \mathcal{K}^{\prime}$.

Again, our notion of bi-interpretability is stronger than strictly necessary, and for many applications it suffices to assume that the mapping $\iota: M \rightarrow \Psi \circ \Phi(M)$ is uniformly definable.

Theorem A.9 Let $\Phi: \mathcal{L}_{0} \rightarrow \mathcal{L}_{1}$ and $\Psi: \mathcal{L}_{1} \rightarrow \mathcal{L}_{0}$ be two interpretation schemes, and let $\mathcal{K}$ and $\mathcal{K}^{\prime}$ be classes of $\mathcal{L}_{0}$ - and of $\mathcal{L}_{1}$-structures, respectively. Assume moreover that $\mathcal{K}$ and $\mathcal{K}^{\prime}$ are bi-interpretable via $(\Phi, \Psi)$.

(i) The class $\mathcal{K}$ is elementary if and only if $\mathcal{K}^{\prime}$ is.

(ii) Assume that for each atomic $\mathcal{L}_{1}$-formula $\varphi$, the definable predicate $\varphi^{\Phi}$ is constant in $\mathcal{K}$, and similarly that $\varphi^{\Psi}$ is constant in $\mathcal{K}^{\prime}$ for every atomic $\mathcal{L}_{0}$ formula $\varphi$. Then $\mathcal{K}$ is model complete (respectively, inductive) if and only if $\mathcal{K}^{\prime}$ is.

(iii) Assume that for each atomic $\mathcal{L}_{1}$-formula $\varphi$, the definable predicate $\varphi^{\Phi}$ is quantifier-free, and similarly that $\varphi^{\Psi}$ is quantifier-free for every atomic $\mathcal{L}_{0}$ formula $\varphi$ (we say that $\Phi$ and $\Psi$ are quantifier-free, or that $\mathcal{K}$ and $\mathcal{K}^{\prime}$ are quantifier-free bi-interpretable). Then $\mathcal{K}$ eliminates quantifiers if and only if $\mathcal{K}^{\prime}$ does.

Proof Assume that $\mathcal{K}=\operatorname{Mod}(T)$. Let $T^{\prime}$ be the theory consisting of $\Psi^{-1}(T)$ along with all the axioms of the form $\varphi(\bar{x})=\varphi^{\Phi \circ \Psi}(\bar{x})$, where $\varphi$ varies over atomic $\mathcal{L}_{1}$ formulae. Clearly, if $N \in \mathcal{K}^{\prime}$ then $N \vDash T^{\prime}$. Conversely, assume that $N \vDash T^{\prime}$. Then $N \vDash \Psi^{-1}(T)$, so $\Psi(N) \in \mathcal{K}$. Thus $\Phi \circ \Psi(N) \in \mathcal{K}^{\prime}$, and the second group of axioms ensures that $N=\Phi \circ \Psi(N)$. Thus $\mathcal{K}^{\prime}=\operatorname{Mod}\left(T^{\prime}\right)$ is elementary, proving the first item (by symmetry).

For the second item, the assumption tells us that if $M_{0} \subseteq M_{1}$ are both in $\mathcal{K}$ then $\Phi\left(M_{0}\right) \subseteq \Phi\left(M_{1}\right)$ in $\mathcal{K}^{\prime}$, and similarly in the direction from $\mathcal{K}^{\prime}$ to $\mathcal{K}$. So assume first 
that $\mathcal{K}$ is model complete and let $N_{0} \subseteq N_{1}$ in $\mathcal{K}^{\prime}$. Then $\Psi\left(N_{0}\right) \subseteq \Psi\left(N_{1}\right)$ in $\mathcal{K}$, so $\Psi\left(N_{0}\right) \preceq \Psi\left(N_{1}\right)$ and thus $N_{0}=\Phi \circ \Psi\left(N_{0}\right) \preceq \Phi \circ \Psi\left(N_{1}\right)=N_{1}$. Assume now that $\mathcal{K}$ is inductive and let $\left\{N_{i}\right\}_{i<\alpha}$ be an increasing chain in $\mathcal{K}^{\prime}$. Then $\left\{\Psi\left(N_{i}\right)\right\}_{i<\alpha}$ is an increasing chain in $\mathcal{K}$, so $M=\bigcup \Psi\left(N_{i}\right) \in \mathcal{K}$ (this being a union of complete structures, i.e., the metric completion of the set union). In particular, $M \supseteq \Psi\left(N_{i}\right)$ for each $i$, so $\Phi(M) \supseteq \Phi \circ \Psi\left(N_{i}\right)=N_{i}$, i.e., $\Phi(M) \supseteq \bigcup N_{i}$. We now use the fact that the $\mathcal{L}_{0}$-definable predicate $d_{\mathcal{L}_{1}}(x, y)^{\Phi}$ is necessarily uniformly continuous, and that the set union of $\Psi\left(N_{i}\right)$ is dense in $M$ (both with respect to $d_{\mathcal{L}_{0}}$ ) to conclude that the set union of the $N_{i}$ is dense in $\Phi(M)$. Considering the complete structure union we have $\bigcup N_{i}=\Phi(M) \in \mathcal{K}^{\prime}$, as desired.

We now turn to the last item. The assumption tells us that if $\varphi$ is any quantifier-free $\mathcal{L}_{0}$-formula, or even a quantifier-free $\mathcal{L}_{0}$-definable predicate, then $\varphi^{\Psi}$ is quantifier-free as well, and similarly in the other direction. Assume $\mathcal{K}$ eliminates quantifiers, and let $\varphi(\bar{x})$ be and $\mathcal{L}_{1}$-formula. Then $\varphi^{\Phi}$ is equivalent in $\mathcal{K}$ to a quantifier-free definable predicate, say $\psi(\bar{x})$, and $\psi^{\Psi}(\bar{x})$ is quantifier-free as well. It will be enough to show that $\psi^{\Psi}$ coincides with $\varphi$ in $\mathcal{K}^{\prime}$. Indeed, let $N=\Phi \circ \Psi(N) \in \mathcal{K}^{\prime}, \bar{a} \in N$. Then

$$
\varphi(\bar{a})^{N}=\varphi(\bar{a})^{\Phi \circ \Psi(N)}=\varphi^{\Phi}(\bar{a})^{\Psi(N)}=\psi(\bar{a})^{\Psi(N)}=\psi^{\Psi}(\bar{a})^{N} .
$$

This completes the proof.

\section{B A convergence rate for approximations of the modular functional}

We conclude with a result that was used in earlier versions of this paper in Section 3 , later superseded by a more direct approach. We chose to keep it here since it is relatively easy and does provide some uniformity for approximations of Nakano spaces by ones in which the essential range of $p$ is finite. Such uniformity may come in handy for an explicit axiomatisation of Nakano spaces, which, at the time of writing, does not yet exist in the literature.

A naïve manner to try to approximate the modular functional is by $\Theta(f) \approx \sum\left\|f_{k}\right\|^{p_{k}}$ where $f=\sum f_{k}$ consists of cutting the domain of $f$ into chunks such that the exponent function $p(\cdot)$ is almost constant $p_{k}$ on each chunk. We show here that these approximations do converge to $\Theta(f)$ at a uniform rate: the difference is always smaller than $C \sqrt{\Delta}$ where $\Delta$ is the maximum of diameters of the range of $p$ on the chunks and $C$ is a constant. 
Lemma B.1 Let $(N, \Theta)=L_{p(\cdot)}(X, \mathfrak{B}, \mu)$, and assume that ess rng $p \subseteq[s, s+\varepsilon]$ where $1 \leq s<s+\varepsilon \leq r$. Let $f \in N$, and assume that $\|f\| \leq 1$. Then $\left|\Theta(f)-\|f\|^{s+\varepsilon}\right| \leq$ $\frac{\varepsilon}{s}|\ln \Theta(f)| \Theta(f)$.

Proof We may assume that $f \geq 0$ and $\|f\|>0$. Let $a=\|f\|$, so $\Theta(f / a)=1$, and for all $t$ :

$$
a^{t}=a^{t}\left(\int(f / a)^{p} d \mu\right)=\int f^{p} a^{t-p} d \mu
$$

Notice that for all $x$ we have $s-p(x) \leq 0 \Longrightarrow a^{s-p(x)} \geq 1$ while $s+\varepsilon-p(x) \geq 0 \Longrightarrow$ $a^{s+\varepsilon-p(x)} \leq 1$, so:

$$
a^{s+\varepsilon}=\int f^{p} a^{s+\varepsilon-p} d \mu \leq \int f^{p} d \mu \leq \int f^{p} a^{s-p} d \mu=a^{s} .
$$

In other words: $a^{s+\varepsilon} \leq \Theta(f) \leq a^{s}$. It follows that $\Theta(f)^{1+\frac{\varepsilon}{s}} \leq a^{s+\varepsilon} \leq \Theta(f)$, whereby

$$
\left|\Theta(f)-a^{s+\varepsilon}\right| \leq\left|\Theta(f)-\Theta(f)^{1+\frac{\varepsilon}{s}}\right| \leq \frac{\varepsilon}{s}|\ln \Theta(f)| \Theta(f),
$$

as desired.

Lemma B.2 There is a constant $C$ such that for every $0<n \in \mathbb{N}$ and every sequence $\left(a_{k}: k<\omega\right)$ such that $a_{k} \geq 0$ and $\sum a_{k} \leq 1$ :

$$
\sum \frac{a_{k}\left|\ln a_{k}\right|}{k+n} \leq \frac{C}{\sqrt{n}}, \quad(0 \ln 0=0) .
$$

Proof At first let us assume that $a_{k} \leq \frac{1}{e}$ for all $k$, noting that $\theta(x)=-x \ln x$ is strictly increasing on $\left[0, \frac{1}{e}\right]$.

We may assume that the sequence is ordered so that $a_{k}\left|\ln a_{k}\right|$ is decreasing. It follows that $\left(a_{k}: k<\omega\right)$ is a decreasing sequence. Since $\sum a_{k} \leq 1$ we have $a_{k} \leq \frac{1}{k+1}<\frac{1}{e}$ for all $k \geq 2$, whereby $a_{k}\left|\ln a_{k}\right| \leq \frac{\ln (k+1)}{k+1}$. Let $C_{0}=\sum \frac{\ln (k+3)}{(k+3)^{3 / 2}}<\infty$. Then:

$$
\sum_{k \geq 2} \frac{a_{k}\left|\ln a_{k}\right|}{k+n} \leq \sum_{k \geq 2} \frac{\ln (k+1)}{(k+n)(k+1)} \leq \frac{1}{\sqrt{n}} \sum_{k \geq 2} \frac{\ln (k+1)}{(k+1)^{3 / 2}}=\frac{C_{0}}{\sqrt{n}} .
$$

In this calculation we ignored the first two terms of the sum. In addition, in the general case there may be at most 2 indexes $k$ such that $a_{k}>\frac{1}{e}$. Together these account for at most $\frac{4}{e n} \leq \frac{4}{e \sqrt{n}}$. Thus $\sum \frac{a_{k}\left|\ln a_{k}\right|}{k+n} \leq \frac{C}{\sqrt{n}}$ where $C=C_{0}+\frac{4}{e}$. 
Lemma B.3 Let $(N, \Theta)=L_{p(\cdot)}(X, \mathfrak{B}, \mu)$ be a Nakano space and let $0<n<\omega$ be fixed. Let $\ell>n(r-1)$, and for $k<\ell$ let $K_{k}=\left[\frac{n+k}{n}, \frac{n+k+1}{n}\right), X_{k}=p^{-1}\left(K_{k}\right)$. Let $C$ be the constant from Lemma B.2.

Then every $f \in N$ can be expressed as $f=\sum_{k<\ell} f_{k}$ where $f_{k}=\left.f\right|_{X_{k}} \in$ $L_{p \Upsilon_{X_{k}}(\cdot)}\left(X_{k}, \mathfrak{B} \uparrow_{X_{k}}, \mu \uparrow_{X_{k}}\right)$. If $\|f\| \leq 1$ then we have:

$$
\left|\Theta_{p(\cdot)}(f)-\sum_{k<\ell}\left\|f_{k}\right\|^{\frac{n+k+1}{n}}\right| \leq \frac{C}{\sqrt{n}} .
$$

Proof We have $\sum_{k<\ell} \Theta\left(f_{k}\right)=\Theta(f) \leq 1$, whereby

$$
\begin{aligned}
\left|\Theta(f)-\sum_{k<\ell}\left\|f_{k}\right\|^{\frac{n+k+1}{n}}\right| & \leq \sum_{k<\ell}\left|\Theta\left(f_{k}\right)-\right|\left|f_{k} \|^{\frac{n+k+1}{n}}\right| \\
& \leq \sum_{k<\ell} \frac{1 / n}{(n+k) / n}\left|\ln \left(\Theta\left(f_{k}\right)\right)\right| \Theta\left(f_{k}\right) \\
& =\sum_{k<\ell} \frac{1}{n+k}\left|\ln \left(\Theta\left(f_{k}\right)\right)\right| \Theta\left(f_{k}\right) \leq \frac{C}{\sqrt{n}},
\end{aligned}
$$

as desired.

\section{References}

[1] I Ben Yaacov, Definability of groups in $\aleph_{0}$-stable metric structures, arXiv: 0802.4286 , submitted for publication.

[2] I Ben Yaacov, On perturbations of continuous structures, arXiv:0802.4388, submitted for publication.

[3] I Ben Yaacov, Topometric spaces and perturbations of metric structures, Logic and Analysis 1 (2008) 235-272, doi: 10.1007/s11813-008-0009-x, arXiv:0802.4458.

[4] I Ben Yaacov, A Berenstein, CW Henson, Model-theoretic independence in the Banach lattices $L^{p}(\mu)$, submitted for publication..

[5] I Ben Yaacov, A Berenstein, C W Henson, A Usvyatsov, Model theory for metric structures, from: "Model theory with Applications to Algebra and Analysis, volume 2", (Z Chatzidakis, D Macpherson, A Pillay, A Wilkie, editors), London Math Society Lecture Note Series 350 (2008) 315-427.

[6] I Ben Yaacov, A Usvyatsov, Continuous first order logic and local stability, Trans. American Math. Soc., to appear, arXiv:0801.4303. 
[7] DH Fremlin, Measure Theory Volume 2: Further Topics in the General Theory, Torres Fremlin, 25 Ireton Road, Colchester CO3 3AT, England (2003), http://www.essex.ac.uk/maths/staff/fremlin/mt2.2003/index.htm.

[8] DH Fremlin, Measure Theory Volume 3: Measure Algebras, Torres Fremlin, 25 Ireton Road, Colchester CO3 3AT, England (2004), http://www.essex.ac.uk/maths/staff/fremlin/mt3.2004/index.htm.

[9] H V Machado, A characterization of convex subsets of normed spaces, Kōdai Mathematical Seminar Reports 25 (1973) 307-320.

[10] P Meyer-Nieberg, Banach Lattices, Springer-Verlag (1991).

[11] L P Poitevin, Model Theory of Nakano Spaces, PhD thesis, University of Illinois at Urbana-Champaign (2006).

[12] L P Poitevin, Y Raynaud, Ranges of positive contractive projections in Nakano spaces, Indagationes Mathematicae, to appear.

Itaï Ben Yaacov, Université de Lyon, Université Lyon 1, INSA de Lyon, F-69621, Ecole Centrale de Lyon, CNRS, UMR5208, Institut Camille Jordan, 43 blvd du 11 novembre 1918, F-69622 Villeurbanne-Cedex, France

http://math.univ-lyon1.fr/〜begnac/

Received: 30 October $2007 \quad$ Revised: 3 October 2008 